\title{
The Geometric Traveling Salesman Problem in the Heisenberg Group
}

Fausto Ferrari, Bruno Franchi and Hervé Pajot

\begin{abstract}
In the Heisenberg group $\mathbb{H}$ (endowed with its Carnot-Carathéodory structure), we prove that a compact set $E \subset \mathbb{H}$ which satisfies an ana$\log$ of Peter Jones' geometric lemma is contained in a rectifiable curve. This quantitative condition is given in terms of Heisenberg $\beta$ numbers which measure how well the set $E$ is approximated by Heisenberg straight lines.
\end{abstract}

\section{Introduction}

Let $(X, d)$ be a metric space. The geometric traveling salesman problem could be stated as follows: Under which (quantitative) conditions is a compact subset $E \subset X$ contained in a rectifiable curve of $X$ (that is a curve of finite length)? Moreover, we would like also to control the length of the shortest curve $\Gamma$ containing $E$ (if such a curve exists). The Euclidean case has been considered by P. Jones [18] who introduced in [17] the $\beta$ numbers. Let $E$ be a compact subset of the Euclidean space $\left(\mathbb{R}^{n}, d_{\text {Euc }}\right)$. For any $x \in \mathbb{R}^{n}$ and any $t>0$, set

$$
\beta_{\text {Euc }}(x, t)=\inf _{L} \sup _{y \in E \cap B(x, t)} \frac{d_{\text {Euc }}(y, L)}{t}
$$

where the infimum is taken over all straight lines $L$ in $\mathbb{R}^{n}$. The $\beta$ numbers measure how well the set $E$ is approximated by straight lines at each point and each scale. Set

$$
\beta_{\mathrm{Euc}}(E)=\int_{\mathbb{R}^{n}} \int_{0}^{+\infty} \beta_{\mathrm{Euc}}(x, t)^{2} d x \frac{d t}{t^{n}}
$$

(where $d x$ is the integration with respect to Lebesgue measure).

2000 Mathematics Subject Classification: 28A75.

Keywords: Heisenberg group, Carnot-Carathéodory metric, rectifiable curve, Traveling Salesman Problem. 
Theorem 1.1. ([18], [21]) Let $E \subset \mathbb{R}^{n}$ be a compact set. Then, $E$ is contained in a rectifiable curve $\Gamma$ if and only if $\beta_{\mathrm{Euc}}(E)<+\infty$. Moreover,

$$
\inf _{\Gamma \supset E} l(\Gamma) \leq C\left(\operatorname{diam} E+\beta_{\mathrm{Euc}}(E)\right)
$$

(where $C>0$ is an absolute constant depending only on $n$ ).

Applications of this result to harmonic measures, limit sets of Kleinian groups, Brownian motion, complex dynamics, singular integral operators are given in [4], [5], [6], [14], [17]. The main goal of this paper is to extend Theorem 1.1 to sub-Riemannian spaces, more precisely to Heisenberg groups. It should be mentionned that a version of the geometric traveling salesman theorem in general metric spaces is given in [16]. In this general setting, $\beta$ numbers are replaced by the Menger curvature (see the Appendix). However, in the Heisenberg group, we can define $\beta$ numbers by using Heisenberg straight lines and prove an exact counterpart of Theorem 1.1. Our interest for this problem is basically of geometric nature, being meant to understand more precisely the geometry of curves in the Heisenberg group, in the spirit e.g. of [3], [15], [1], [11], [12]. Nevertheless, the problem attacked here can be seen as the most simple example of the following situation: consider a mechanical non-holonomic system $X$ (a "robot"), and let $E \subset X$ be a set of configurations. We seek conditions on $E$ that guarantee that we can move the system through all the these configurations in a finite time. Before stating our main results, we establish some notations. More details are given in Section 2.

Let $\mathbb{H}$ be the (first) Heisenberg group endowed with its Carnot-Carathéodory metric $d_{c}$. The points in $\mathbb{H}$ will be denoted as $P=[z, t]=[x+i y, t]$. If $P=[z, t], Q=[\zeta, \tau] \in \mathbb{H}$, following the notations of [27], we define the group operation

$$
P \cdot Q=[z+\zeta, t+\tau+2 \Im m(z \bar{\zeta})] .
$$

For $s \geq 0$, denote by $\mathcal{H}_{c}^{s}$ the $s$-dimensional Hausdorff measure associated with $d_{c}$. In this paper, we shall call straight $\mathbb{H}$-line any set $\ell$ of the form $\ell=a \cdot r$, where $a$ is a point of $\mathbb{H}$ and $r$ is any Euclidean straight line through the origin lying in the set $\{[z, 0] z \in \mathbb{C}\}$. We shall denote by $G(\mathbb{H}, 1)$ the set of all straight $\mathbb{H}$-lines. In addition, we denote by $G_{0}(\mathbb{H}, 1)$ the set of all Euclidean straight lines through the origin lying in the set $\{[z, 0] z \in \mathbb{C}\}$, i.e. $r \in G_{0}(\mathbb{H}, 1)$ if and only if $r=\left\{\left(\left[\lambda e^{i \omega}, 0\right], \lambda \in \mathbb{R}\right\}\right.$.

Remark 1.1. Seeking an intrinsic notion of straight line we are naturally lead to look for laterals of subgroups that are in addition invariant under dilations and have intrinsic Hausdorff dimension equal to 1. It follows from [12] that $G(\mathbb{H}, 1)$ is in fact the good choice. 
An important metric feature of the elements of $G(\mathbb{H}, 1)$ is that $d$ is additive on $r \in G(\mathbb{H}, 1)$.

Let $E \subset \mathbb{H}$ be a compact set, and let $P \in \mathbb{H}$. For $t>0$, we set

$$
\beta_{\mathbb{H}}(P, t)=\inf _{L \in G(\mathbb{H}, 1)} \sup _{Q \in E \cap B_{c}(P, t)} \frac{d_{c}(Q, L)}{t} .
$$

Here, $B_{c}(P, t)$ denotes the closed ball (with respect to $d_{c}$ ) whose center is $P$ and whose radius is $t$. Set

$$
\beta_{\mathbb{H}}(E)=\int_{\mathbb{H}} \int_{0}^{+\infty} \beta_{\mathbb{H}}(P, t)^{2} d P \frac{d t}{t^{4}},
$$

where $d P$ is the integration with respect to the Hausdorff measure $\mathcal{H}_{c}^{4}$ of $\left(\mathbb{H}, d_{c}\right)$. Note that the exponent 4 in $\mathcal{H}_{c}^{4}$ and in $\frac{d t}{t^{4}}$ is the Hausdorff dimension of $\left(\mathbb{H}, d_{c}\right)$.

Our main result is an analog of theorem 1.1 in the Heisenberg group.

Theorem 1.2. Let $E \subset \mathbb{H}$ be a compact set. Then, $E$ is contained in a rectifiable curve $\Gamma$ if $\beta_{\mathbb{H}}(E)<+\infty$. Moreover,

$$
\inf _{\Gamma \supset E} l(\Gamma) \leq C\left(\operatorname{diam} E+\beta_{\mathbb{H}}(E)\right)
$$

(where $C>0$ is an absolute constant)

The construction of the curve $\Gamma$ is based on the algorithm given in [18] (see also [22]). To estimate $l(\Gamma)$, the main ingredient in [18] is the Pythagorean theorem. In the Heisenberg group, such result does not exist. Our idea is to compare Euclidean triangles and Carnot-Carathéodory triangles. This yields to a curvature type estimate for the Heisenberg group that we now explain.

Let $(X, d)$ be a connected length space. A triangle is a collection of three points (= vertices) $x, y, z$ of $X$ connected by three shortest paths (= sides), denoted by $(x, y),(y, z)$ and $(z, x)$. For such triangle, a comparison triangle is a triangle in the Euclidean plane, with vertices $\bar{x}, \bar{y}, \bar{z}$ such that

$$
d(x, y)=|\bar{x}-\bar{y}| ; d(y, z)=|\bar{y}-\bar{z}| ; d(z, x)=|\bar{z}-\bar{x}| .
$$

We say that the metric space $(X, d)$ is a space of nonnegative curvature if in some neighborhood of each point the following holds:

For every triangle with vertices $x, y, z$ and every point $w \in(x, y)$, one has $d(z, w) \geq d(\bar{z}, \bar{w})$ where $\bar{w}$ is is the point on the side $[\bar{x}, \bar{y}]$ of a comparison triangle with vertices $\bar{x}, \bar{y}, \bar{z}$ such that $d(w, x)=|\bar{w}-\bar{x}|$. 
This condition means that comparison Euclidean triangles are thinner than triangles in $X$. Following [8, chapter 10], an Alexandrov space is a metric space with nonnegative curvature. For Alexandrov spaces, Hausdorff dimension equals topological dimension. But, the Hausdorff dimension of $\left(\mathbb{H}, d_{c}\right)$ is 4 whereas its topological dimension is 3 (see below). Thus, the Heisenberg group is not an Alexandrov space. However, for some particular triangles (that is "almost equilateral"), comparison Euclidean triangles are thinner than triangles in the Heisenberg group. More precisely, we have the

Theorem 1.3. Suppose $P_{1}, P_{2}, P_{3}$ are points in $\mathbb{H}$ such that

$$
P_{1} \text { and } P_{2} \text { belong to } r_{0} \in G(\mathbb{H}, 1)
$$

and there exist $c_{i, j}>0, C_{i, j}>0$ for $i, j=1,2,3, i \neq j$ such that for some $t>0$

$$
c_{i, j} t \leq d\left(P_{i}, P_{j}\right) \leq C_{i, j} t
$$

In addition denote by $\tilde{P}_{i}, i=1,2,3$ three points in the Euclidean space $\mathbb{R}^{2}$ such that

$$
d_{\text {Euc }}\left(\tilde{P}_{i}, \tilde{P}_{j}\right)=d_{c}\left(P_{i}, P_{j}\right) \quad i, j=1,2,3,
$$

and by $\tilde{r}_{0}$ the straight line through $\tilde{P}_{1}$ and $\tilde{P}_{2}$. Then there exists $c=$ $c\left(c_{i, j}, C_{i, j}\right)>0$ independent of $t>0$ such that

$$
d_{c}\left(P_{3}, r_{0}\right) \geq c d_{\text {Euc }}\left(\tilde{P}_{3}, \tilde{r}_{0}\right) .
$$

From this comparison theorem and the classical Pythagorean theorem in the complex plane, we get (with the same assumptions)

$$
d_{c}\left(P_{1}, P_{3}\right)+d_{c}\left(P_{2}, P_{3}\right)-d_{c}\left(P_{1}, P_{2}\right) \leq C \frac{d_{c}\left(P_{3}, r_{0}\right)^{2}}{t}
$$

This estimate (as in the Euclidean case) is crucial to get a bound of the length of the curve $\Gamma$. In fact, we will also prove that (1.4) remains true without the assumption that $P_{1}$ and $P_{2}$ belong to the same straight line $r_{0}$.

It is natural to ask whether the condition

$$
\int_{\mathbb{H}} \int_{0}^{1} \beta_{\mathbb{H}}^{2}(P, t) d P \frac{d t}{t^{4}}<\infty .
$$

is necessary. In other words, to ask whether any compact set $E$ contained in a rectifiable curve $\gamma$ satisfies (1.5). In the Euclidean case, this has been proved by P. Jones [18] by using complex variables methods for $n=2$ and by K. Okikiolu [21] for any $n$ with constants depending on the dimension (see theorem 1.1). Recently, R. Schul [26] has considered the case of (infinite dimensionnal) Hilbert spaces. 
The sub-Riemannian case we consider in this paper is quite different from these situations. We will prove that for subsets of regular curves in the Heisenberg group, (1.5) holds. More precisely, we have the

Theorem 1.4. For any $\mathbf{C}^{1, \alpha}$-regular simple horizontal curve $\gamma$ we have

$$
\int_{\mathbb{H}} \int_{0}^{1} \beta_{\mathbb{H}}(P, t)^{2} d P \frac{d t}{t^{4}}<\infty .
$$

In particular, (1.6) holds for geodesics.

Notice that the horizontality assumption cannot be dropped: a rectifiable Lipschitz continuous curve is horizontal (see for instance [24] or [25]), hence a non-horizontal curve cannot be contained in a rectifiable curve. Note also that a geodesic curve is smooth (see [13] or [1] and [3] where explicit equations of geodesics are given).

More details concerning the Heisenberg group and its Carnot-Carathéodory structure are given in the next section where the reader will find the proof of Theorem 1.3. Section 3 is dedicated to the construction of the curve $\Gamma$. The proof of Theorem 1.4 is given in Section 4. In an Appendix, we collect some useful facts concerning rectifiable curves in general metric spaces.

\section{Some geometric results concerning the Heisenberg group}

\subsection{Background concerning Heisenberg groups}

In this paper we denote by $\mathbb{H}$ the 1-dimensional Heisenberg group $\mathbb{H}^{1} \simeq$ $\mathbb{C} \times \mathbb{R} \simeq \mathbb{R}^{3}$. Points in $\mathbb{H}$ will be written as $P=[z, t]=[x+i y, t]$. If $P=[z, t], Q=[\zeta, \tau] \in \mathbb{H}$ and $r>0$, following the notations of [27], we define the group operation

$$
P \cdot Q=[z+\zeta, t+\tau+2 \Im m(z \bar{\zeta})]
$$

and the family of non isotropic dilations $\delta_{r}$ defined as

$$
\delta_{r}(P):=\left[r z, r^{2} t\right]
$$

It is also useful to consider the group translations $\tau_{P}: \mathbb{H} \rightarrow \mathbb{H}$ defined as

$$
Q \mapsto \tau_{P}(Q):=P \cdot Q
$$

for any fixed $P \in \mathbb{H}$. We remind that $P^{-1}:=[-z,-t]$ is the inverse of $P$. 
We endow $\mathbb{H}$ with the homogeneous norm

$$
\|P\|=\left(\|z\|^{4}+|t|^{2}\right)^{1 / 4}=\left(\left(z_{1}^{2}+z_{2}^{2}\right)^{2}+t^{2}\right)^{1 / 4}
$$

and define the gauge distance (Korányi's distance) as

$$
d(P, Q):=\left\|P^{-1} \cdot Q\right\|
$$

We explicitly observe that

Proposition 2.1. The function $d$ defined by (2.1) is a distance in $\mathbb{H}$ (see [27, p.638]) and the usual properties related with translations and dilations hold, i.e. for any $P, Q, Q^{\prime} \in \mathbb{H}$ and for any $r>0$

$$
d\left(\tau_{P} Q, \tau_{P} Q^{\prime}\right)=d\left(Q, Q^{\prime}\right) \quad \text { and } \quad d\left(\delta_{r} Q, \delta_{r} Q^{\prime}\right)=\operatorname{rd}\left(Q, Q^{\prime}\right) .
$$

In addition, for any bounded subset $\Omega$ of $\mathbb{H}$ there exist positive constants $c_{1}(\Omega), c_{2}(\Omega)$ such that

$$
c_{1}(\Omega)|P-Q|_{\mathbb{R}^{3}} \leq d(P, Q) \leq c_{2}(\Omega)|P-Q|_{\mathbb{R}^{3}}^{1 / 2}
$$

for $P, Q \in \Omega$. In particular, the topologies defined by $d$ and by the Euclidean distance coincide on $\mathbb{H}$.

Remark 2.1. We stress that, because the topologies defined by d and by the Euclidean distance coincide, the topological dimension of $\mathbb{H}$ is 3 . On the contrary the Hausdorff dimension of $(\mathbb{H}, d)$ is $Q=4$ (see e.g. [20]).

From now on, $U(P, r)$ and $B(P, r)$ will be, respectively, the open and closed balls with center $P$ and radius $r$ with respect to the distance $d$. We notice explicitly that $U(P, r)$ is an Euclidean smooth domain in $\mathbb{R}^{3}$.

We remind also that Lebesgue measure $d \mathcal{L}^{3}=d z d t$ in $\mathbb{C} \times \mathbb{R}$ is left (and right) invariant under group translations and hence it is the Haar measure of the group.

If $s \geq 0$, following Federer [10, Section 2.10], denote by $\mathcal{H}_{d}^{s}$ the $s$-dimensional Hausdorff measure obtained from the distance $d$ in $\mathbb{H}$. Recall that, for any subset $A \subset \mathbb{H}$, its Hausdorff measure $H_{d}^{s}(A)$ (with respect to $d$ ) is defined by

$$
H_{d}^{s}(A)=\lim _{\delta \rightarrow 0}\left(\inf \left\{\sum_{i \in I} \alpha(s) 2^{-s}\left(\operatorname{diam} A_{i}\right)^{s} ; \operatorname{diam} A_{i}<\delta, A \subset \cup_{i \in I} A_{i}\right\}\right),
$$

where

$$
\alpha(s)=\Gamma\left(\frac{1}{2}\right)^{s} / \Gamma\left(\frac{s}{2}+1\right) .
$$

For further results about Hausdorff measures, we refer to [10]. Translation invariance and homogeneity under dilations of Hausdorff measures follow as usual from (2.2). More precisely we have 
Proposition 2.2. Let $A \subseteq \mathbb{H}, P \in \mathbb{H}$. Then, if $s \geq 0$,

$$
\begin{gathered}
\mathcal{H}_{d}^{s}\left(\tau_{P} A\right)=\mathcal{H}_{d}^{s}(A) \\
\mathcal{H}_{d}^{s}\left(\delta_{r}(A)\right)=r^{s} \mathcal{H}_{d}^{s}(A) .
\end{gathered}
$$

The Lie algebra of the left invariant vector fields of $\mathbb{H}^{n}$ is generated by

$$
X=\frac{\partial}{\partial x}+2 y \frac{\partial}{\partial t}, \quad Y=\frac{\partial}{\partial y}-2 x \frac{\partial}{\partial t} ; \quad T=\frac{\partial}{\partial t}
$$

and the only non-trivial commutation relation is

$$
[X, Y]=-4 T
$$

In the following we shall identify vector fields and associated first order differential operators. The vector fields $X, Y$ define a vector bundle on $\mathbb{H}$ (the horizontal vector bundle $\mathrm{HH}$ ) that can be canonically identified with a vector subbundle of the tangent vector bundle of $\mathbb{R}^{3}$. Since each fiber of $H \mathbb{H}$ can be canonically identified with a two-dimensional vector subspace of $\mathbb{R}^{3}$, each section $\phi$ of $\mathrm{H} \mathbb{H}$ can be identified with a map $\phi: \mathbb{H} \rightarrow \mathbb{R}^{3}$. At each point $P \in \mathbb{H}$ the horizontal fiber is indicated as $\mathrm{HH}_{P}$ and each fiber can be endowed with the scalar product $\langle\cdot, \cdot\rangle_{P}$ and the norm $|\cdot|_{P}$ that make the vector fields $X, Y$ orthonormal. Hence we shall also identify a section of $\mathrm{HHI}$ with its canonical coordinates with respect to this moving frame. In this way, a section $\phi$ will be identified with a function $\phi: \mathbb{H} \rightarrow \mathbb{R}^{2}$. As it is common in Riemannian geometry, when dealing with two sections $\phi$ and $\psi$ whose argument is not explicitly written, we shall drop the index $P$ in the scalar product writing $\langle\psi, \phi\rangle$ for $\langle\psi(P), \phi(P)\rangle_{P}$. The same convention shall be adopted for the norm.

We say also that an absolutely continuous curve $\gamma:[0, T] \rightarrow \mathbb{H}$ is horizontal if $\gamma^{\prime}$ is a horizontal section, i.e. if $\gamma^{\prime}(t) \in \mathrm{HH}_{\gamma(t)}$ for a.e, $t \in[0, T]$.

We can remind now the notion of Carnot-Carathéodory distance (ccdistance) in $\mathbb{H}$.

Definition 2.3. If $P, Q \in \mathbb{H}$, their cc-distance $d_{c}(P, Q)$ is defined by

$$
d_{c}(P, Q)=\inf \left\{\int_{0}^{T}\left|\gamma^{\prime}(t)\right|_{\gamma(t)} d t\right\}
$$

where the infimum is taken over all horizontal curves $\gamma:[0, T] \rightarrow \mathbb{H}$ such that $\gamma(0)=P$ and $\gamma(T)=Q$.

Open (respectively closed) balls with respect to $d_{c}$ will be denoted by $U_{c}(x, r)$ and $B(x, r)$. 
It is well known $([28])$ that

Proposition 2.4. There exist $\alpha_{1}, \alpha_{2}>0$ such that

$$
\alpha_{1} d(P, Q) \leq d_{c}(P, Q) \leq \alpha_{2} d(P, Q)
$$

for all $P, Q \in \mathbb{H}$.

Lemma 2.5. The distance $d$ is invariant under cylindrical rotations of the form

$$
[z, t] \rightarrow\left[e^{i \theta} z, t\right], \quad \theta \in \mathbb{R}
$$

Proof. It is enough to notice that

$$
\left[e^{i \theta} \zeta, \tau\right]^{-1}\left[e^{i \theta} z, t\right]=\left[e^{i \theta}(z-\zeta), t-\tau-2 \Im m\left(e^{i \theta} \zeta e^{-i \theta} \bar{z}\right)\right] .
$$

From now on, we call straight $\mathbb{H}$-line any set $\ell$ of the form $\ell=a \cdot r$, where $a$ is a point of $\mathbb{H}$ and $r$ is any Euclidean straight line through the origin lying in the set $\{[z, 0] z \in \mathbb{C}\}$. We shall denote by $G(\mathbb{H}, 1)$ the set of all straight $\mathbb{H}$-lines. In addition, we denote by $G_{0}(\mathbb{H}, 1)$ the set of all Euclidean straight line through the origin lying in the set $\{[z, 0] z \in \mathbb{C}\}$, i.e. $r \in G_{0}(\mathbb{H}, 1)$ if and only if $r=\left\{\left(\left[\lambda e^{i \omega}, 0\right], \lambda \in \mathbb{R}\right\}\right.$.

In a more intrinsic way, $r \in G_{0}(\mathbb{H}, 1)$ if and only if $r$ is both a subgroup of $\mathbb{H}$ that is dilation invariant and a 1-dimensional $\mathbb{H}$-regular submanifold (see [12]). The elements of $r \in G(\mathbb{H}, 1)$ are laterals of the elements of $r \in G_{0}(\mathbb{H}, 1)$.

An important metric feature of the elements of $G(\mathbb{H}, 1)$ is that $d$ is additive on $r \in G(\mathbb{H}, 1)$.

Lemma 2.6. Let $r \in G(\mathbb{H}, 1)$ be given, and let $P_{1}, P_{2}, P_{3}$ be successive points in $r$. Then $d\left(P_{1}, P_{3}\right)=d\left(P_{1}, P_{2}\right)+d\left(P_{2}, P_{3}\right)$.

Proof. In fact, it is enough to check this property for $r \in G_{0}(\mathbb{H}, 1)$, since the distance is invariant under left translations. Thus, assume $P_{i}=\left[\lambda_{i} e^{i \omega}, 0\right]$, $i=1,2,3$, with $\lambda_{1}<\lambda_{2}<\lambda_{3}$. It is enough to show that

$$
d\left(P_{i}, P_{j}\right)=\left|\lambda_{i}-\lambda_{j}\right|
$$

Indeed we have (keeping into account that $\left.\Im m\left(\lambda_{j} e^{i \omega}, \overline{\lambda_{i} e^{i \omega}}\right)\right]=0$ )

$$
d\left(P_{i}, P_{j}\right)=\left\|\left[-\lambda_{j} e^{i \omega}, 0\right] \cdot\left[\lambda_{i} e^{i \omega}, 0\right]\right\|=\left\|\left[\left(\lambda_{i}-\lambda_{j}\right) e^{i \omega}, 0\right]\right\|=\left|\lambda_{i}-\lambda_{j}\right|,
$$

and we are done.

We now give an other property of our straight lines. 
Lemma 2.7. If $y \in L$ with $L=a \cdot r, r \in G_{0}(\mathbb{H}, 1)$, then $L=y \cdot r$.

Proof. If $r=\left\{\left(\left[\lambda e^{i \omega}, 0\right], \lambda \in \mathbb{R}\right\}\right.$, by assumption, $y=a \cdot w, w=\left[\lambda e^{i \omega}, 0\right]$ for $\lambda \in \mathbb{R}$ suitable, so that $y^{-1} \cdot a=w^{-1}=\left[-\lambda e^{i \omega}, 0\right]$. If $z$ is any point in $L$, then analogously $z=a \cdot\left[\mu e^{i \omega}, 0\right]$ for $\mu \in \mathbb{R}$ suitable. Then

$$
z=y \cdot y^{-1} \cdot a \cdot\left[\mu e^{i \omega}, 0\right]=y \cdot\left[-\lambda e^{i \omega}, 0\right] \cdot\left[\mu e^{i \omega}, 0\right]=y \cdot\left[(\mu-\lambda) e^{i \omega}, 0\right] .
$$

Since $\left[(\mu-\lambda) e^{i \omega}, 0\right] \in r$, the proof is complete.

Definition 2.8. We say that a curve $\gamma:[0, T] \rightarrow \mathbb{H}$ is a minimal geodesic (a segment) if $d_{c}(\gamma(t), \gamma(\tau))=|t-\tau|$ for all $t, \tau \in[0, T]$.

Since $\mathbb{H}$ endowed with the distance $d_{c}$ is a complete and locally compact metric length space in the sense of [15], Definition 1.7, then any two points of $\mathbb{H}$ can be joined by (at least) a minimal geodesic ([15], Theorem 1.10). If $P, Q \in \mathbb{H}$, we denote by $[P, Q]$ a minimal geodesic connecting $P$ and $Q$. This notion is not mesleading, since, if $P, Q \in \ell \in G(\mathbb{H}, 1)$, then $[P, Q]$ is precisely the segment of $\ell$ with endpoints $P$ and $Q$.

Let $E \subset \mathbb{H}$ be a compact set, and let $P \in \mathbb{H}$. For $t>0$, we set

$$
\beta_{\mathbb{H}}(P, t)=\inf _{L \in G(\mathbb{H}, 1)} \sup _{Q \in E \cap B_{c}(P, t)} \frac{d_{c}(Q, L)}{t} .
$$

\subsection{A curvature type estimate}

The main goal of this section is to prove the following statement. As a corollary, we will get theorem 1.3.

Theorem 2.9. Let $\epsilon_{0} \in[0,1)$ be given. Then there exists $c=c\left(\epsilon_{0}\right)>0$ such that, if $P_{1}, P_{2}, P_{3}$ are points in $\mathbb{H}$ such that

$$
P_{1} \text { and } P_{2} \text { belong to } r_{0} \in G(\mathbb{H}, 1) \text {, }
$$

and in addition we denote by $Q_{i}, i=1,2,3$ the points in the Euclidean space $\mathbb{R}^{2}$ such that

$$
d_{\mathrm{Euc}}\left(Q_{i}, Q_{j}\right)=d\left(P_{i}, P_{j}\right) \quad i, j=1,2,3,
$$

and by $\tilde{r}_{0}$ the straight line through $Q_{1}$ and $Q_{2}$, then

$$
d\left(P_{3}, r_{0}\right) \geq c d_{\text {Euc }}\left(Q_{3}, \tilde{r}_{0}\right) .
$$


Proof. By triangle inequality and (2.6), $d\left(P_{1}, P_{2}\right) \leq 2 d\left(P_{1}, P_{3}\right)$, and we may assume $d\left(P_{1}, P_{2}\right)<2 d\left(P_{1}, P_{3}\right)$, since otherwise the point $Q_{3}$ would lie on $\tilde{r}_{0}$, so that $d_{\text {Euc }}\left(Q_{3}, \tilde{r}_{0}\right)=0$, and the assertion would hold trivially.

By Lemma 2.7, $r_{0}=y \cdot r$, with $r=\left\{\left(\left[\lambda e^{i \omega}, 0\right], \lambda \in \mathbb{R}\right\} \in G_{0}(\mathbb{H}, 1)\right.$. Moreover, by Lemma 2.5, we can assume also $\omega=0$. Since the distance is invariant under group translations, without loss of generality, we can assume $r_{0}=r$ and $P_{1}=-P_{2}$.

Thanks to the dilations of the group, we may rescale the picture so that

$$
1=d\left(P_{1}, P_{3}\right) \geq d\left(P_{2}, P_{3}\right) \geq(1-\epsilon) .
$$

Let us consider first the case $d\left(P_{1}, P_{3}\right)=d\left(P_{2}, P_{3}\right)=1$.

We can write $P_{1}=[\alpha / 2+i 0,0], P_{2}=[-\alpha / 2+i 0,0]$, with $1 \leq \alpha<2$ (since $1=d\left(P_{1}, P_{3}\right) \leq d\left(P_{1}, P_{2}\right) \leq 2 d\left(P_{1}, P_{3}\right)=2$ and $\left.d\left(P_{1}, P_{2}\right)=1\right)$, and $P_{3}=\left[z_{1}+i z_{2}, z_{3}\right]$. Hence $r_{0}=\{[\eta+i 0,0], \eta \in \mathbb{R}\}$. Since $d\left(P_{1}, P_{2}\right)=$ $d\left(P_{2}, P_{3}\right)=1$, we have

$$
\left(z_{1}^{2}+z_{2}^{2}+\frac{\alpha^{2}}{4}-\alpha z_{1}\right)^{2}+z_{3}^{2}+2 \alpha z_{3} z_{2}+\alpha^{2} z_{2}^{2}=1
$$

and

$$
\left(z_{1}^{2}+z_{2}^{2}+\frac{\alpha^{2}}{4}+\alpha z_{1}\right)^{2}+z_{3}^{2}-2 \alpha z_{3} z_{2}+\alpha^{2} z_{2}^{2}=1 .
$$

Subtracting we get

$$
z_{3} z_{2}=z_{1}\left(z_{1}^{2}+z_{2}^{2}+\frac{\alpha^{2}}{4}\right)
$$

and then adding

$$
\left(z_{1}^{2}+z_{2}^{2}+\frac{\alpha^{2}}{4}\right)^{2}+\alpha^{2} z_{1}^{2}+z_{3}^{2}+\alpha^{2} z_{2}^{2}=1
$$

We assume also $z_{i} \neq 0, i=1,2,3$. Replacing (2.10) in (2.11) we obtain that (2.10) and (2.11) imply

$$
\left\{\begin{array}{l}
z_{3} z_{2}=z_{1}\left(z_{1}^{2}+z_{2}^{2}+\frac{\alpha^{2}}{4}\right) \\
\left(\frac{z_{2} z_{3}}{z_{1}}\right)^{2}+\alpha^{2} z_{1}^{2}+z_{3}^{2}+\alpha^{2} z_{2}^{2}=1
\end{array}\right.
$$

i.e.

$$
\left\{\begin{array}{c}
z_{3} z_{2}=z_{1}\left(z_{1}^{2}+z_{2}^{2}+\frac{\alpha^{2}}{4}\right) \\
\left(z_{3}^{2}+\alpha^{2} z_{1}^{2}\right)\left(z_{1}^{2}+z_{2}^{2}\right)=z_{1}^{2}
\end{array}\right.
$$


Putting now $z_{1}=\rho \cos \theta, z_{2}=\rho \sin \theta, \theta \in(0,2 \pi), \theta \neq k \frac{\pi}{2}, k=1,2,3$ (the last restriction will be dropped soon), formula (2.13) becomes

$$
\left\{\begin{array}{l}
z_{3}=\frac{\cos \theta}{\sin \theta}\left(\rho^{2}+\frac{\alpha^{2}}{4}\right), \\
\rho^{2}\left(z_{3}^{2}+\alpha^{2} \rho^{2} \cos ^{2} \theta\right)=\rho^{2} \cos ^{2} \theta .
\end{array}\right.
$$

After some elementary algebraic manipulations we get

$$
\left\{\begin{array}{l}
z_{3}^{2}+\alpha^{2} \sin \theta \cos \theta z_{3}-\left(1+\frac{\alpha^{4}}{4}\right) \cos ^{2} \theta=0 \\
\rho^{2}=\frac{\cos ^{2} \theta-z_{3}^{2}}{\alpha^{2} \cos ^{2} \theta}
\end{array}\right.
$$

Since our aim to provide a lower bound for the distance of $P_{3}$ from $r_{0}$ when $P_{3}$ satisfy (2.8) and (2.9), it is enough to estimate the same quantity when

$$
z_{3}=\frac{1}{2}\left(-\alpha^{2} \sin \theta \pm \sqrt{\alpha^{4} \sin ^{2} \theta+\alpha^{4}+4}\right) \cos \theta:=\frac{1}{2} \beta \cos \theta, \quad 0 \leq \theta \leq 2 \pi .
$$

In addition, because of the second identity in (2.15), we must restrict ourselves to those choices of $\theta$ and of the sign \pm in $\beta$ such that $|\beta|=|\beta(\theta)| \leq 2$. This implies that $\beta$ can be written better as follows

$$
\beta=\left\{\begin{array}{l}
-\alpha^{2} \sin \theta+\sqrt{\alpha^{4} \sin ^{2} \theta+\alpha^{4}+4}, \text { when } \sin \theta>0 \\
-\alpha^{2} \sin \theta-\sqrt{\alpha^{4} \sin ^{2} \theta+\alpha^{4}+4}, \text { when } \sin \theta<0 .
\end{array}\right.
$$

In particular

$$
|\beta|=\sqrt{\alpha^{4} \sin ^{2} \theta+\alpha^{4}+4}-\alpha^{2}|\sin \theta| .
$$

Replacing then (2.16) in (2.15) we get

$$
\begin{gathered}
\rho^{2}=\frac{1}{\alpha^{2}}\left(1-\frac{\beta^{2}}{4}\right), \\
z_{1}=\frac{\cos \theta}{\alpha} \sqrt{1-\frac{\beta^{2}}{4}} \text { and } z_{2}=\frac{\sin \theta}{\alpha} \sqrt{1-\frac{\beta^{2}}{4}} \quad 0 \leq \theta \leq 2 \pi .
\end{gathered}
$$

If $\eta \in \mathbb{R}$, we put $\psi_{\theta}(\eta):=d(z,(\eta, 0,0))^{4}$, where $z=\left(z_{1}, z_{2}, z_{3}\right)$ satisfies $(2.16)$ and (2.20). Hence

$$
\psi_{\theta}(\eta)=\left(\rho^{2}-2 \eta \rho \cos \theta+\eta^{2}\right)^{2}+\left(\frac{\beta}{2} \cos \theta+2 \eta \rho \sin \theta\right)^{2}=I_{1}^{2}+I_{2}^{2},
$$

where

$$
I_{1}=\rho^{2}-2 \eta \rho \cos \theta+\eta^{2}, \quad I_{2}=\frac{\beta}{2} \cos \theta+2 \eta \rho \sin \theta .
$$


We want to provide a lower bound of

$$
m:=\inf _{\eta \in \mathbb{R}} \inf _{0 \leq \theta \leq 2 \pi} \psi_{\theta}(\eta)
$$

In addition, we can restrict ourselves to assume $|\eta| \leq 2 \alpha$, since e.g. for $\eta>2 \alpha$ we have

$d(z,(\eta, 0,0)) \geq d\left(\left(\frac{\alpha}{2}, 0,0\right),(\eta, 0,0)\right)-d\left(\left(\frac{\alpha}{2}, 0,0\right), z\right)=\left|\frac{\alpha}{2}-\eta\right|-1 \geq \frac{3 \alpha}{2}>1$.

The strategy of the proof is the following one: first we check that $\psi_{\theta}$ is convex. This implies that

$$
\psi_{\theta}(\eta) \geq \psi_{\theta}(0)+\eta \psi_{\theta}^{\prime}(0)
$$

Indeed

$$
\frac{1}{2} \psi_{\theta}^{\prime \prime}(\eta)=(-2 \rho \cos \theta+2 \eta)^{2}+4 \rho^{2} \sin ^{2} \theta+2\left(\rho^{2}-2 \eta \rho \cos \theta+\eta^{2}\right) \geq 0,
$$

since $\rho^{2}-2 \eta \rho \cos \theta+\eta^{2} \geq \rho^{2}-2|\eta \rho|+\eta^{2}=(|\rho|-|\eta|)^{2}$.

The next step consists of proving the following lemma.

Lemma 2.10. There exists $c_{1}>0$ such that

$$
\eta \psi_{\theta}^{\prime}(0) \geq-c_{1} \psi_{\theta}(\eta)-\frac{1}{2} \psi_{\theta}(0)
$$

Combining (2.22) and Lemma 2.10, it follows easily that there exists $c_{2}>0$ such that

$$
\psi_{\theta}(\eta) \geq c_{2} \psi_{\theta}(0)
$$

for $\eta \in \mathbb{R}$ and $0 \leq \theta \leq 2 \pi$.

Finally, we prove the following lemma.

Lemma 2.11. There exist $c_{3}>0$ (independent of $\alpha$ ) such that

$$
\psi_{\theta}(0) \geq c_{3}\left(1-\frac{\alpha^{2}}{4}\right)^{2} .
$$

In such a way, we have proved the following estimate.

Proposition 2.12. There exists $c_{4}>0$ (independent of $\alpha$ ) such that

$$
d\left(P_{3}, r_{0}\right) \geq c_{4} \sqrt{1-\frac{\alpha^{2}}{4}}
$$


Proof of Lemma 2.10. We have

$$
\psi_{\theta}^{\prime}(\eta)=2 I_{1}(-2 \rho \cos \theta+2 \eta)+4 I_{2} \rho \sin \theta
$$

and hence

$$
\psi_{\theta}^{\prime}(0)=4 \rho\left(\frac{\beta}{2} \sin \theta \cos \theta-\rho^{2} \cos \theta\right) .
$$

First, we want to show that

$$
|\eta| \rho^{3} \leq c\left(I_{1}^{2}+I_{2}^{2}\right)=c \psi_{\theta}(\eta)
$$

or, equivalently,

$$
\left|\frac{\eta}{\rho}\right|^{2} \leq c \frac{1}{\rho^{4}}\left(I_{1}^{2}+I_{2}^{2}\right)=c\left[\left(1-2 \frac{\eta}{\rho} \cos \theta+\frac{\eta^{2}}{\rho^{2}}\right)^{2}+\left(\frac{\beta}{2 \rho^{2}} \cos \theta+2 \frac{\eta}{\rho} \sin \theta\right)^{2}\right] \text {. }
$$

Let $\epsilon_{1}$ be a positive number close to zero that will be explicitly chosen later (see (2.29)), and distinguish two cases:

i) $|\cos \theta|<1-\epsilon_{1}$ :

ii) $|\cos \theta| \geq 1-\epsilon_{1}$.

Case i). In this case

$$
\frac{1}{\rho^{2}} I_{1}=1-2 \frac{\eta}{\rho} \cos \theta+\frac{\eta^{2}}{\rho^{2}} \geq 1-2\left(1-\epsilon_{1}\right)\left|\frac{\eta}{\rho}\right|+\frac{\eta^{2}}{\rho^{2}} \geq 2 \epsilon_{1}\left|\frac{\eta}{\rho}\right|
$$

since for any $b \in \mathbb{R}$ we have $1-2\left(1-\epsilon_{1}\right) b+b^{2}-2 \epsilon_{1} b>0$. Thus in this case (2.26) is proved.

Case ii). In this case $|\sin \theta| \leq \sqrt{1-\left(1-\epsilon_{1}\right)^{2}} \leq \sqrt{2 \epsilon_{1}}$. Then

$$
\frac{1}{\rho^{2}}\left|I_{2}\right| \geq\left|\frac{|\beta|}{2 \rho^{2}}\right| \cos \theta\left|-2 \frac{|\eta|}{\rho}\right| \sin \theta||=\frac{\alpha}{\rho}\left|\frac{|\beta|}{2 \alpha \rho}\right| \cos \theta\left|-2 \frac{|\eta|}{\alpha}\right| \sin \theta|| .
$$

Notice now that, by (2.16),

$$
\begin{aligned}
|\beta| & \geq \sqrt{\alpha^{4} \sin ^{2} \theta+\alpha^{4}+4}-\alpha^{2}|\sin \theta|=\frac{\alpha^{4}+4}{\sqrt{\alpha^{4} \sin ^{2} \theta+\alpha^{4}+4}+\alpha^{2}|\sin \theta|} \\
& \geq \frac{\alpha^{4}+4}{2 \sqrt{\alpha^{4} \sin ^{2} \theta+\alpha^{4}+4}} \geq \frac{\alpha^{4}+4}{2 \sqrt{2\left(\alpha^{4}+4\right)}}=\frac{\sqrt{\alpha^{4}+4}}{2 \sqrt{2}} \geq \sqrt{\frac{5}{8}}
\end{aligned}
$$

since $\alpha \geq 1$. 
Now, by (2.19), keeping into account that the function $t \rightarrow \frac{t}{\sqrt{4-t^{2}}}$ is increasing on $(0,2)$, we have

$$
\begin{aligned}
\frac{|\beta|}{2 \alpha \rho}|\cos \theta| & =\frac{|\beta|}{2 \sqrt{1-\beta^{2} / 4}}|\cos \theta|=\frac{|\beta|}{\sqrt{4-\beta^{2}}}|\cos \theta| \\
& \geq \frac{\sqrt{\frac{5}{8}}}{\sqrt{4-5 / 8}}|\cos \theta|:=\gamma_{0}|\cos \theta| \geq\left(1-\epsilon_{1}\right) \gamma_{0},
\end{aligned}
$$

by ii). On the other hand

$$
2 \frac{|\eta|}{\alpha}|\sin \theta| \leq 4|\sin \theta| \leq 4 \sqrt{2 \epsilon_{1}}<\frac{1}{2}\left(1-\epsilon_{1}\right) \gamma_{0},
$$

provided we choose $\epsilon_{1}$ sufficiently small. Thus, going back to (2.28), we get

$$
\frac{1}{\rho^{2}}\left|I_{2}\right| \geq \frac{\alpha}{2 \rho}\left(1-\epsilon_{1}\right) \gamma_{0} \geq \frac{|\eta|}{4 \rho}\left(1-\epsilon_{1}\right) \gamma_{0} .
$$

Recall that $\eta \leq 2 \alpha$ (see above).

Thus, combining (2.27) and (2.30), (2.26) follows with

$$
c=\min \left\{\frac{1}{4}\left(1-\epsilon_{1}\right) \gamma_{0}, \epsilon_{1}\right\}^{-2} .
$$

The next step consists of proving that there exists $C>0$ such that

$$
J:=\left|4 \rho \eta \frac{\beta}{2} \sin \theta \cos \theta\right| \leq \frac{1}{2} \psi_{\theta}(0)+C \psi_{\theta}(\eta)
$$

An elementary argument and (2.26) yield

$$
\begin{aligned}
J & \leq \frac{1}{2} \frac{\beta^{2}}{4} \cos ^{2} \theta+8 \eta^{2} \rho^{2} \sin ^{2} \theta \leq \frac{1}{2} \frac{\beta^{2}}{4} \cos ^{2} \theta+8 \eta^{2} \rho^{2} \leq \frac{1}{2} \psi_{\theta}(0)+8 \eta^{2} \rho^{2} \\
& =\frac{1}{2} \psi_{\theta}(0)+8 \rho^{4}\left(\frac{\eta}{\rho}\right)^{2} \\
& \leq \frac{1}{2} \psi_{\theta}(0)+8 \rho^{4} \min \left\{\frac{1}{4}\left(1-\epsilon_{1}\right) \gamma_{0}, \epsilon_{1}\right\}^{-2} \frac{1}{\rho^{4}}\left(I_{1}^{2}+I_{2}^{2}\right) \\
& =\frac{1}{2} \psi_{\theta}(0)+8 \min \left\{\frac{1}{4}\left(1-\epsilon_{1}\right) \gamma_{0}, \epsilon_{1}\right\}^{-2} \psi_{\theta}(\eta)
\end{aligned}
$$

and (2.31) follows.

Combining eventually (2.24), (2.25) and (2.31), Lemma 2.10 is completely proved. 
Proof of Lemma 2.11. Let us write $\delta:=t \sqrt{1-\frac{\alpha^{2}}{4}}$, where $t>0$ will be fixed later, and distinguish two cases:

i) $|\beta| \leq 2 \sqrt{1-\alpha^{2} \delta^{2}}$.

ii) $|\beta|>2 \sqrt{1-\alpha^{2} \delta^{2}}$;

Case i). In this case (remember (2.19))

$$
\psi_{\theta}(0)=\rho^{4}+\frac{\beta^{2}}{4} \cos ^{2} \theta \geq \rho^{4}=\frac{1}{\alpha^{4}}\left(1-\frac{\beta^{2}}{4}\right)^{2} \geq \delta^{4}=t^{4}\left(1-\frac{\alpha^{2}}{4}\right)^{2}
$$

Case ii). By (2.18),

$$
\sqrt{\alpha^{4} \sin ^{2} \theta+\alpha^{4}+4}>\alpha^{2}|\sin \theta|+2 \sqrt{1-\alpha^{2} \delta^{2}}
$$

and hence

$$
\alpha^{4} \sin ^{2} \theta+\alpha^{4}+4>\alpha^{4} \sin ^{2} \theta+4\left(1-\alpha^{2} \delta^{2}\right)+4 \alpha^{2}|\sin \theta| \sqrt{1-\alpha^{2} \delta^{2}},
$$

that yields

$$
\alpha^{4}+4 \alpha^{2} \delta^{2}>4 \alpha^{2}|\sin \theta| \sqrt{1-\alpha^{2} \delta^{2}}
$$

and eventually

$$
|\sin \theta|<\frac{\alpha^{2}+4 \delta^{2}}{4 \sqrt{1-\alpha^{2} \delta^{2}}}
$$

Thus

$$
|\cos \theta|>\sqrt{1-\frac{\left(\alpha^{2}+4 \delta^{2}\right)^{2}}{16\left(1-\alpha^{2} \delta^{2}\right)}}=\frac{\sqrt{16\left(1-\alpha^{2} \delta^{2}\right)-\left(\alpha^{2}+4 \delta^{2}\right)^{2}}}{4 \sqrt{1-\alpha^{2} \delta^{2}}} .
$$

Now a direct computation shows that

$$
\begin{aligned}
16\left(1-\alpha^{2} \delta^{2}\right) & -\left(\alpha^{2}+4 \delta^{2}\right)^{2}=4\left(1-\frac{\alpha^{2}}{4}\right)\left(\alpha^{2}+4-6 \alpha^{2} t^{2}+t^{4} \alpha^{2}-4 t^{4}\right) \\
& =4\left(1-\frac{\alpha^{2}}{4}\right)\left(\alpha^{2}+4-t^{2}\left(t^{2}\left(4-\alpha^{2}\right)+6 \alpha^{2}\right)\right) \geq 16\left(1-\frac{\alpha^{2}}{4}\right),
\end{aligned}
$$

provided we can choose $t>0$ sufficiently small but independent of $\alpha$ such that

$$
\alpha^{2}+4-t^{2}\left(t^{2}\left(4-\alpha^{2}\right)+6 \alpha^{2}\right)>4
$$

By the way, this is possible since $1 \leq \alpha \leq 2$.

Then, in case ii), we have the estimate

$$
\psi_{\theta}(0)=\rho^{4}+\frac{\beta^{2}}{4} \cos ^{2} \theta \geq \frac{\beta^{2}}{4} \cos ^{2} \theta \geq\left(1-\frac{\alpha^{2}}{4}\right)^{2} .
$$

Combining (2.33) and (2.34) we achieve the proof of Lemma 2.11. 
To achieve now the proof of the Theorem we have but to get rid of the assumption $d\left(P_{2}, P_{3}\right)=1$. Thus, we assume $d\left(P_{2}, P_{3}\right)=1-\epsilon$. If we denote by $\left[P_{1}, P_{2}\right]$ the segment $\left\{[s, 0],-\frac{\alpha}{2} \leq s \leq \frac{\alpha}{2}\right\}$, we can distinguish two cases:

i) $d\left(P_{3}, Q\right) \geq 1-\epsilon$ for any $Q \in\left[P_{1}, P_{2}\right]$;

ii) there exists $Q^{\prime} \in\left[P_{1}, P_{2}\right]$ such that $d\left(P_{3}, Q^{\prime}\right)<1-\epsilon$.

Suppose first case i) holds. Then, let us show that there exists $c_{5}>0$ (independent of $\epsilon$ and $\alpha$ ) such that

$$
d\left(P_{3}, r_{0}\right) \geq c_{5}(1-\epsilon)
$$

Notice first that the $d$-ball $B\left(P_{3}, 1-\epsilon\right)$ is a closed convex set (in the standard sense), since $B(0,1-\epsilon)$ is convex and group translations carry (Euclidean) straight lines into (Euclidean) straight lines. Thus, keeping into account that $P_{2} \in \partial B\left(P_{3}, 1-\epsilon\right)$ and that $\partial B\left(P_{3}, 1-\epsilon\right)$ does not contain segments, we can conclude that either $r_{0} \cap B\left(P_{3}, 1-\epsilon\right)=\left\{P_{2}\right\}$ and then (2.35) is proved with $c_{5}=1$, or there exists $Q_{1}=\left[\frac{\alpha}{2}+h, 0\right], h>0$ such that $r_{0} \cap B\left(P_{3}, 1-\epsilon\right)=$ $\left[P_{2}, Q_{1}\right]$. Moreover, by triangle inequality, $h<2(1-\epsilon)$. Now, by continuity of $d\left(P_{3}, \cdot\right)$, necessarily $d\left(P_{3}, Q_{1}\right)=1-\epsilon$. Rescaling the picture of a factor $1 /(1-\epsilon)$, the points $P_{2}, Q_{1}$, and $P_{3}$ satisfy the assumption of Proposition 2.12 with $\alpha=h /(1-\epsilon)$. Thus

$$
d\left(P_{3}, r_{0}\right) \geq c_{4}(1-\epsilon) \sqrt{1-\frac{h^{2}}{4(1-\epsilon)^{2}}} .
$$

On the other hand, if we consider the points $P_{1}, Q_{1}$ and $P_{3}$, by triangle inequality we get $d\left(P_{1}, Q_{1}\right) \leq 2-\epsilon$. But the distance is additive in $r_{0}$, so that $d\left(P_{1}, Q_{1}\right)=\alpha+h$, and hence $h \leq 2-\alpha-\epsilon$. Replacing in (2.36), we get

$$
d\left(P_{3}, r_{0}\right) \geq c_{4}(1-\epsilon) \sqrt{1-\frac{(2-\alpha-\epsilon)^{2}}{4(1-\epsilon)^{2}}} .
$$

But $\alpha \geq 1$, and hence $2-\alpha-\epsilon \leq 1-\epsilon$. Therefore (2.35) holds with

$$
c_{5}=c_{4} \sqrt{\frac{3}{4}}
$$

Finally, keeping in mind that $\alpha \leq 2-\epsilon$ by triangle inequality, by (2.35) we get that, if i) holds, then

$$
c_{6}(1-\epsilon) \sqrt{1-\frac{\alpha^{2}}{(2-\epsilon)^{2}}} \leq d\left(P_{3}, r_{0}\right) .
$$


We can consider now the case ii). By continuity, there exists $Q \in$ $\left[P_{1}, Q^{\prime}\right] \subset\left[P_{1}, P_{2}\right]$ such that $d\left(P_{3}, Q\right)=1-\epsilon=d\left(P_{3}, P_{2}\right)$. Rescaling again the picture of a factor $1 /(1-\epsilon)$, the points $Q, P_{2}, P_{3}$ satisfy the assumption of Proposition 2.12 with $\alpha=d\left(Q, P_{2}\right) /(1-\epsilon)$, and then we get

$$
d\left(P_{3}, r_{0}\right) \geq c(1-\epsilon) \sqrt{1-\frac{d\left(Q, P_{2}\right)^{2}}{4(1-\epsilon)^{2}}} .
$$

On the other hand, by triangle inequality, $d\left(P_{1}, Q\right) \geq d\left(P_{3}, P_{1}\right)-d\left(P_{3}, Q\right)=\epsilon$, and hence, keeping in mind that the distance in $r_{0}$ is additive, $d\left(Q, P_{2}\right)=$ $d\left(P_{1}, P_{2}\right)-d\left(P_{1}, Q\right) \leq \alpha-\epsilon$. Thus

$$
\frac{d\left(Q, P_{2}\right)^{2}}{4(1-\epsilon)^{2}} \leq \frac{(\alpha-\epsilon)^{2}}{4(1-\epsilon)^{2}}<\frac{\alpha^{2}}{(2-\epsilon)^{2}} \leq 1
$$

since

$$
(2-\epsilon)(\alpha-\epsilon)-2 \alpha(1-\epsilon)=\epsilon(\alpha+\epsilon-2)<0,
$$

because $\alpha<2-\epsilon$, by triangle inequality. Replacing then (2.39) in (2.38), we get eventually

$$
d\left(P_{3}, r_{0}\right) \geq c(1-\epsilon) \sqrt{1-\frac{\alpha^{2}}{(2-\epsilon)^{2}}} .
$$

In other words, (2.37) still holds also in case ii). Notice that Proposition 2.12 is but a particular case of the above statement when $\epsilon=0$.

We can achieve now the proof of Theorem 2.9 providing an estimate from above of $d_{\text {Euc }}\left(Q_{3}, \tilde{r}_{0}\right)$. Again, we can rescale the picture assuming that $d_{\text {Euc }}\left(Q_{1}, Q_{3}\right)=1$, and putting $d_{\text {Euc }}\left(Q_{2}, Q_{3}\right)=1-\epsilon$ and $d_{\text {Euc }}\left(Q_{1}, Q_{2}\right)=\alpha$. We stress that the rescaling does not affect the final result, since both $d$ and $d_{\text {Euc }}$ are homogeneous of degree one under their respective intrinsic dilations, and the rescaling factor $d\left(P_{1} . P_{3}\right)$ in the Heisenberg group equals by definition the rescaling factor $d_{\text {Euc }}\left(Q_{1}, Q_{3}\right)$ in the Euclidean plane.

We can obtain $d_{\text {Euc }}\left(Q_{3}, \tilde{r}_{0}\right)$ using Hero's theorem as follows

$$
d_{\mathrm{Euc}}\left(Q_{3}, \tilde{r}_{0}\right)=\frac{1}{2 \alpha} \sqrt{(2+\alpha-\epsilon)(\alpha-\epsilon)(\alpha+\epsilon)(2-\alpha-\epsilon)} .
$$

On the other hand $(\alpha-\epsilon)(\alpha+\epsilon) \leq \alpha^{2}$, and hence

$$
\begin{aligned}
d_{\text {Euc }}\left(Q_{3}, \tilde{r}_{0}\right) & \leq \frac{1}{2} \sqrt{(2+\alpha-\epsilon)(2-\alpha-\epsilon)}=\frac{2-\epsilon}{2} \sqrt{1-\frac{\alpha^{2}}{(2-\epsilon)^{2}}} \\
& \leq \sqrt{1-\frac{\alpha^{2}}{(2-\epsilon)^{2}}} \leq \frac{1}{c\left(1-\epsilon_{0}\right)} d\left(P_{3}, r_{0}\right)
\end{aligned}
$$

by (2.40) and (2.37). This completes the proof of Theorem 2.9. 
We now can prove theorem 1.3 for the Heisenberg group equiped with the metric $d$. The case of the Carnot-Carathéodory metric $d_{c}$ follows easily by proposition 2.4 (see also the discussion at the end of section 2.3).

Proof. Without loss of generality we may assume that

$$
d\left(P_{1}, P_{3}\right) \geq d\left(P_{2}, P_{3}\right)
$$

Suppose first

$$
d\left(P_{1}, P_{2}\right) \geq d\left(P_{1}, P_{3}\right) \geq d\left(P_{2}, P_{3}\right)
$$

We apply Theorem 2.9. To this end, we notice that

$$
d\left(P_{2}, P_{3}\right) \geq c_{2,3} t=\frac{c_{2,3}}{C_{1,3}} C_{1,3} t \geq \frac{c_{2,3}}{C_{1,3}} d\left(P_{1}, P_{3}\right)
$$

By (2.42), $\frac{c_{2,3}}{C_{1,3}} \leq 1$. Therefore we can set $\epsilon_{0}=1-\frac{c_{2,3}}{C_{1,3}}$ and then we apply Theorem 2.9. Thus we are left with the case

$$
d\left(P_{1}, P_{3}\right) \geq d\left(P_{2}, P_{3}\right) \quad \text { and } \quad d\left(P_{1}, P_{2}\right) \leq d\left(P_{1}, P_{3}\right)
$$

We can rescale the picture assuming $t=1$. An elementary argument in the Euclidean plane shows that in this case $0<c_{1} \leq d_{\text {Euc }}\left(\tilde{P}_{3}, \tilde{r}_{0}\right) \leq c_{2}$, where $c_{1}, c_{2}$ are absolute geometric constants depending only on $c_{i, j}$ and $C_{i, j}, i,=1,2,3$. Thus, we must prove that there exists a geometric constant $c_{3}>0$ (independent of $P_{i}, i=1,2,3$ ) such that

$$
d\left(P_{3}, r_{0}\right) \geq c_{3}
$$

By contradiction, suppose (2.44) fails to hold. Then for any $n \in \mathbb{N}$ there exist $P_{i}^{n}$ such that

$$
\begin{gathered}
P_{1}^{n} \text { and } P_{2}^{n} \text { belong to } r_{0}^{n} \in G(\mathbb{H}, 1), \\
d\left(P_{1}^{n}, P_{3}^{n}\right) \geq d\left(P_{2}^{n}, P_{3}^{n}\right), \\
c_{i, j} \leq d\left(P_{i}^{n}, P_{j}^{n}\right) \leq C_{i, j}
\end{gathered}
$$

where $c_{i, j}$ and $C_{i, j}$ are the constants of (1.2), and

$$
d\left(P_{3}^{n}, r_{0}^{n}\right)<\frac{1}{n}
$$


Since (2.45)-(2.48) are invariant under group translations, without loss of generality we may assume that $P_{3}^{n} \equiv 0$, and hence that $P_{1}^{n}, P_{2}^{n}$ belong to a compact set, because of (2.47). Thus, we can assume that $P_{i}^{n} \rightarrow P_{i}$ as $n \rightarrow \infty$ for $i=1,2$. By Lemma 2.7, we can assume $r_{0}^{n}=P_{1}^{n} \cdot\left\{\left[\lambda e^{i \omega_{n}}, 0\right]: \lambda \in \mathbb{R}\right\}$, with $\omega_{n} \rightarrow \omega$ as $n \rightarrow \infty$, so that also $P_{2} \in r_{0}:=P_{1} \cdot\left\{\left[\lambda e^{i \omega}, 0\right]: \lambda \in \mathbb{R}\right\}$. By (2.48), for any $n \in \mathbb{N}$ there exists $\lambda_{n} \in \mathbb{R}$ such that $\left[\lambda_{n} e^{i \omega_{n}}, 0\right]=\left(P_{1}^{n}\right)^{-1}$. $Q^{n}$, with $d\left(Q^{n}, 0\right)<\frac{1}{n}$. Again by compactness, we can assume $\lambda_{n} \rightarrow \lambda$ and hence $P_{1} \cdot\left[\lambda e^{i \omega}, 0\right]=0$, i.e. $0 \in r_{0}$. Since $d$ is additive on $r_{0}$ and in addition $d\left(P_{1}, 0\right) \geq d\left(P_{2}, 0\right)$, and $d\left(P_{1}, P_{2}\right) \leq d\left(P_{1}, 0\right)$, then 0 cannot lie between $P_{1}$ and $P_{2}$, and, analogously, $P_{2}$ cannot lie between 0 and $P_{1}$. Thus we get a contradiction, keeping in mind that all these distances do no vanish (they are in fact comparable to 1 ).

Corollary 2.13. Let $P_{1}, P_{2}, P_{3}$ be as in Theorem 1.3. Then there exists $C=C\left(c_{i, j}, C_{i, j}\right)>0$ such that

$$
d\left(P_{1}, P_{3}\right)+d\left(P_{2}, P_{3}\right)-d\left(P_{1}, P_{2}\right) \leq C \frac{d\left(P_{3}, r_{0}\right)^{2}}{t}
$$

Proof. With the notations of Theorem 1.3, an elementary argument in the Euclidean plane shows that

$$
\begin{aligned}
& d\left(P_{1}, P_{3}\right)+d\left(P_{2}, P_{3}\right)-d\left(P_{1}, P_{2}\right) \\
& \quad=d\left(\tilde{P}_{1}, \tilde{P}_{3}\right)+d\left(\tilde{P}_{2}, \tilde{P}_{3}\right)-d\left(\tilde{P}_{1}, \tilde{P}_{2}\right) \leq C d_{\text {Euc }}^{2}\left(\tilde{P}_{3}, \tilde{r}_{0}\right) .
\end{aligned}
$$

Applying (1.3) in Theorem 1.3 we achieve the proof.

We now prove (2.49). For this, let $H$ be the orthogonal projection of $\tilde{P}_{3}$ on $\tilde{r}_{0}$ and set $h=d_{\text {Euc }}\left(\tilde{P}_{3}, \tilde{r}_{0}\right)$. Then, by Pythagorean Theorem, we get

$$
\begin{aligned}
& d_{\text {Euc }}\left(\tilde{P}_{1}, \tilde{P}_{3}\right)+d_{\text {Euc }}\left(\tilde{P}_{2}, \tilde{P}_{3}\right)-d_{\text {Euc }}\left(\tilde{P}_{1}, \tilde{P}_{2}\right) \\
& =\sqrt{d_{\text {Euc }}\left(\tilde{P}_{1}, H\right)^{2}+h^{2}}+\sqrt{d_{\text {Euc }}\left(\tilde{P}_{2}, H\right)+h^{2}}-d_{\text {Euc }}\left(\tilde{P}_{1}, H\right)-d_{\text {Euc }}\left(\tilde{P}_{2}, H\right) \\
& \leq d_{\text {Euc }}\left(\tilde{P}_{1}, H\right)\left(\sqrt{\left.1+\frac{h^{2}}{d_{\text {Euc }}\left(\tilde{P}_{1}, H\right)^{2}}-1\right)+}\right. \\
& \quad+d_{\text {Euc }}\left(\tilde{P}_{2}, H\right)\left(\sqrt{1+\frac{h^{2}}{d_{\text {Euc }}\left(\tilde{P}_{2}, H\right)^{2}}}-1\right) \\
& \leq C \frac{h^{2}}{t} .
\end{aligned}
$$




\subsection{The main estimate}

Let $t>0$; we are now in position to give a sharp upper bound of the quantity

$$
d\left(P_{3}, P_{1}\right)+d\left(P_{3}, P_{2}\right)-d\left(P_{1}, P_{2}\right) .
$$

in terms of

$$
\max \left\{t^{-1} d\left(P_{i}, r_{0}\right): i=1,2,3\right\},
$$

where $r_{0} \in G(\mathbb{H}, 1)$ and $P_{i} \in \mathbb{H}$, are $i=1,2,3$ any three given points at comparable mutual distance with $t$.

Theorem 2.14. Let $r_{0} \in G(\mathbb{H}, 1)$ and $t>0$ be given, and let $P_{1}, P_{2}, P_{3} \in \mathbb{H}$ be such that

i) $d\left(P_{1}, P_{2}\right) \geq d\left(P_{1}, P_{3}\right) \geq d\left(P_{2}, P_{3}\right)$;

ii) there exist $c_{i, j}>0, C_{i, j}>0$ for $i, j=1,2,3, i \neq j$ such that

$$
c_{i, j} t \leq d\left(P_{i}, P_{j}\right) \leq C_{i, j} t
$$

Assume now

$$
\begin{aligned}
\beta:=\max \left\{t^{-1} d\left(P_{i}, r_{0}\right)\right. & : i=1,2,3\} \\
\leq \beta_{0} & :=\frac{1}{6} \min \left\{c_{i, j}, i, j=1,2,3, i \neq j\right\} .
\end{aligned}
$$

Then there exists $C>0, C=C\left(c_{i, j}, C_{i, j}\right)$ such that

$$
d\left(P_{3}, P_{1}\right)+d\left(P_{3}, P_{2}\right)-d\left(P_{1}, P_{2}\right) \leq C t \beta^{2} .
$$

Proof. As usual, taking into account all invariance properties under left translations, without loss of generality we can assume $r_{0}=\{[s, 0]: s \in \mathbb{R}\}$.

By definition, $U\left(P_{i}, 3 \beta_{0} t\right) \cap r_{0} \neq \emptyset$ for $i=1,2,3$, and, in addition, $B\left(P_{i}, 3 \beta_{0} t\right) \cap B\left(P_{j}, 3 \beta_{0} t\right)=\emptyset$ for $i \neq j$, since, if $\xi \in B\left(P_{i}, 3 \beta_{0} t\right)$, then

$$
d\left(\xi, P_{j}\right) \geq d\left(P_{i}, P_{j}\right)-d\left(\xi, P_{i}\right) \geq c_{i, j} t-3 \beta_{0} t \geq\left(6 \beta_{0}-3 \beta_{0}\right) t \geq 3 \beta_{0} t .
$$

Thus, we may choose two couples of points $P_{i A}, P_{i B} \in \partial U\left(P_{i}, 3 \beta_{0} t\right) \cap r_{0}$, $i=1,2$.

In addition, we can assume that, giving an order $<$ on the straight line $r_{0}$, then $P_{1 B}<P_{1 A}<P_{2 A}<P_{2 B}$ since the points can be chosen in such a way the segments $\left[P_{1 B}, P_{1 A}\right]$ and $\left[P_{2 A}, P_{2 B}\right]$ are contained respectively in $B\left(P_{1}, 3 \beta_{0} t\right) \cap r_{0}$ and $B\left(P_{2}, 3 \beta_{0} t\right) \cap r_{0}$ and therefore are disjoint.

The core of the following argument consists of applying successively Corollary 2.13 at the scale $t$ to the triplets of points $\left\{P_{i}, P_{j A}, P_{j B}\right\}, i=1,2,3$, $j=1,2$. 
To this end, we have but to show that

$$
d\left(P_{i}, P_{j A}\right) \approx d\left(P_{i}, P_{j B}\right) \approx d\left(P_{j A}, P_{j B}\right) \approx t,
$$

since $P_{i A}, P_{i B} \in r_{0}$. Indeed, $d\left(P_{i}, P_{i A}\right)=d\left(P_{i}, P_{i B}\right)=3 \beta_{0} t$; on the other hand, there exist $M_{1} \in U\left(P_{1}, 3 \beta_{0} t\right) \cap\left[P_{1 B}, P_{1 A}\right]$ and $M_{2} \in U\left(P_{2}, 3 \beta_{0} t\right) \cap$ $\left[P_{2 A}, P_{2 B}\right]$ such that $d\left(P_{i}, M_{i}\right)=d\left(P_{i}, r_{0}\right) \leq \beta_{0} t, i=1,2$. Keeping in mind that $d$ is additive on $r_{0}$ (Lemma 2.6), we have (for instance)

$$
\begin{aligned}
6 \beta & \geq d\left(P_{1 B}, P_{1 A}\right)=d\left(P_{1 B}, M_{1}\right)+d\left(M_{1}, P_{1 A}\right) \\
& \geq d\left(P_{1 B}, P_{1}\right)-d\left(P_{1}, M_{1}\right)+d\left(P_{1 A}, P_{1}\right)-d\left(P_{1}, M_{1}\right) \geq 4 \beta_{0} t .
\end{aligned}
$$

Moreover,

$$
\begin{aligned}
\left(3 \beta_{0} t+C_{1,3}\right) t & \geq d\left(P_{1 A}, P_{1}\right)+d\left(P_{1}, P_{3}\right) \\
& \geq d\left(P_{1 A}, P_{3}\right) \geq d\left(P_{3}, P_{1}\right)-d\left(P_{1}, P_{1 A}\right) \geq c_{i, j} t-3 \beta_{0} t \geq 3 \beta_{0} t
\end{aligned}
$$

By triangle inequality and keeping in mind Corollary 2.13, we get:

$$
\begin{aligned}
\leq & d\left(P_{1}, P_{1 A}\right)+d\left(P_{1 A}, P_{3}\right)+d\left(P_{2}, P_{3}\right)-d\left(P_{1}, P_{2}\right) \\
= & \left(d\left(P_{1}, P_{1 A}\right)+d\left(P_{1}, P_{1 B}\right)-d\left(P_{1 A}, P_{1 B}\right)\right)-d\left(P_{1}, P_{1 B}\right)+d\left(P_{1 A}, P_{1 B}\right) \\
& +d\left(P_{1 A}, P_{3}\right)+d\left(P_{2}, P_{3}\right)-d\left(P_{1}, P_{2}\right) \\
\leq & C \frac{d^{2}\left(P_{1}, r_{0}\right)}{t}-d\left(P_{1}, P_{1 B}\right)+d\left(P_{1 A}, P_{1 B}\right)+d\left(P_{1 A}, P_{3}\right) \\
& +d\left(P_{2}, P_{3}\right)-d\left(P_{1}, P_{2}\right) \\
\leq & C \frac{d^{2}\left(P_{1}, r_{0}\right)}{t}+d\left(P_{2 A}, P_{3}\right)+d\left(P_{2 A}, P_{2}\right) \\
& -d\left(P_{1}, P_{1 B}\right)+d\left(P_{1 A}, P_{1 B}\right)+d\left(P_{1 A}, P_{3}\right)-d\left(P_{1}, P_{2}\right) \\
= & C \frac{d^{2}\left(P_{1}, r_{0}\right)}{t}+\left(d\left(P_{2 A}, P_{2}\right)+d\left(P_{2 B}, P_{2}\right)-d\left(P_{2 B}, P_{2 A}\right)\right)-d\left(P_{2 B}, P_{2}\right) \\
& +d\left(P_{2 B}, P_{2 A}\right)+d\left(P_{2 A}, P_{3}\right)-d\left(P_{1}, P_{1 B}\right)+d\left(P_{1 A}, P_{1 B}\right) \\
& +d\left(P_{1 A}, P_{3}\right)-d\left(P_{1}, P_{2}\right) \\
\leq & C \frac{d^{2}\left(P_{1}, r_{0}\right)}{t}+C \frac{d^{2}\left(P_{2}, r_{0}\right)}{t}-d\left(P_{2 B}, P_{2}\right)+d\left(P_{2 B}, P_{2 A}\right) \\
& +d\left(P_{2 A}, P_{3}\right)-d\left(P_{1}, P_{1 B}\right)+d\left(P_{1 A}, P_{1 B}\right)+d\left(P_{1 A}, P_{3}\right)-d\left(P_{1}, P_{2}\right) \\
= & C d^{2}\left(P_{1}, r_{0}\right)+C d^{2}\left(P_{2}, r_{0}\right)+\left(d\left(P_{2 A}, P_{3}\right)+d\left(P_{1 A}, P_{3}\right)-d\left(P_{1 A}, P_{2 A}\right)\right) \\
& +d\left(P_{1 A}, P_{2 A}\right)-d\left(P_{2 B}, P_{2}\right)+d\left(P_{2 B}, P_{2 A}\right)-d\left(P_{1}, P_{1 B}\right) \\
& +d\left(P_{1 A}, P_{1 B}\right)-d\left(P_{1}, P_{2}\right) \\
\leq & C \frac{d^{2}\left(P_{1}, r_{0}\right)}{t}+C \frac{d^{2}\left(P_{2}, r_{0}\right)}{t}+C \frac{d^{2}\left(P_{3}, r_{0}\right)}{t}+\left(d\left(P_{1 A}, P_{2 A}\right)+d\left(P_{2 B}, P_{2 A}\right)\right. \\
& \left.+d\left(P_{1 A}, P_{1 B}\right)\right)-\left(d\left(P_{2 B}, P_{2}\right)+d\left(P_{1}, P_{1 B}\right)+d\left(P_{1}, P_{2}\right)\right) . \\
& +d{ }) \\
&
\end{aligned}
$$


Let us recall now that $d$ is additive on $r_{0}$ (Lemma 2.6), so that

$$
d\left(P_{1 A}, P_{2 A}\right)+d\left(P_{2 B}, P_{2 A}\right)+d\left(P_{1 A}, P_{1 B}\right)=d\left(P_{1 B}, P_{2 B}\right) .
$$

On the other hand

$$
d\left(P_{1 B}, P_{2 B}\right) \leq d\left(P_{2 B}, P_{2}\right)+d\left(P_{1}, P_{1 B}\right)+d\left(P_{1}, P_{2}\right)
$$

hence from (2.52) we conclude the proof since

$$
\begin{aligned}
d\left(P_{3}, P_{1}\right)+d\left(P_{3}, P_{2}\right)-d\left(P_{1}, P_{2}\right) & \leq C\left(\frac{d^{2}\left(P_{1}, r_{0}\right)}{t}+\frac{d^{2}\left(P_{2}, r_{0}\right)}{t}+\frac{d^{2}\left(P_{3}, r_{0}\right)}{t}\right) \\
& \leq C^{\prime} \beta^{2} t
\end{aligned}
$$

The proof of Theorem 2.14 relies on Theorem 1.3 via Corollary 2.13, as well as on the fact that $d$ is additive on $r_{0} \in G(\mathbb{H}, 1)$. On the other hand, by Proposition 2.4, Theorem 1.3 still holds when we replace $d$ by the CarnotCarathéodory distance $d_{c}$, and it is easy to see that the same happens for Corollary 2.13. Now, also $d_{c}$ is additive on $r_{0} \in G(\mathbb{H}, 1)$, as we can see by the explicit forms of geodesics in $\mathbb{H}$. Thus, the following theorem holds.

Theorem 2.15. Let $r_{0} \in G(\mathbb{H}, 1)$ and $t>0$ be given, and let $P_{1}, P_{2}, P_{3} \in \mathbb{H}$ be such that

i) $d_{c}\left(P_{1}, P_{2}\right) \geq d_{c}\left(P_{1}, P_{3}\right) \geq d_{c}\left(P_{2}, P_{3}\right)$;

ii) there exist $c_{i, j}>0, C_{i, j}>0$ for $i, j=1,2,3, i \neq j$ such that

$$
c_{i, j} t \leq d_{c}\left(P_{i}, P_{j}\right) \leq C_{i, j} t
$$

Assume now

$$
\begin{aligned}
\beta_{c}:=\max \left\{t^{-1} d_{c}\left(P_{i}, r_{0}\right)\right. & : \quad i=1,2,3\} \\
\leq \beta_{0} & :=\frac{1}{4} \min \left\{c_{i, j}, i, j=1,2,3, i \neq j\right\} .
\end{aligned}
$$

Then there exists $C>0, C=C\left(c_{i, j}, C_{i, j}\right)$ such that

$$
d_{c}\left(P_{3}, P_{1}\right)+d_{c}\left(P_{3}, P_{2}\right)-d_{c}\left(P_{1}, P_{2}\right) \leq C t \beta_{c}^{2} .
$$

Corollary 2.16. Let $P_{1}, P_{2}, P_{3} \in E$ be as in Theorem 2.15, and let $P_{i}$ be a fixed point among them. If $\beta_{\mathbb{H}}\left(P_{i}, C_{0} t\right) \leq \beta_{0} / 2$, with $C_{0} \geq \max \left\{C_{i j}\right\}$, then

$$
d_{c}\left(P_{0}, P_{1}\right)+d_{c}\left(P_{2}, P_{3}\right)-d_{c}\left(P_{1}, P_{2}\right) \leq C t \beta_{\mathbb{H}}\left(P_{i}, C_{0} t\right) .
$$


Proof. By definition, there exists $r_{0} \in G(\mathbb{H}, 1)$ such that

$$
\begin{aligned}
\beta_{c}: & =\max \left\{t^{-1} d_{c}\left(P_{j}, r_{0}\right), j=1,2,3\right\} \\
& \leq \sup _{Q \in E \cap U_{c}\left(P_{i}, C_{0} t\right)} \frac{d_{c}\left(Q, r_{0}\right)}{t} \leq 2 \beta_{\mathbb{H}}\left(P_{i}, C_{0} t\right) \leq \beta_{0},
\end{aligned}
$$

since $P_{j} \in B\left(P_{i}, C_{0} t\right)$ for $i, j=1,2,3$, by ii) and our choice of $C_{0}$.

Thus, we can apply Theorem 2.15, and the assertion follows combining (2.53) and (2.55).

\section{Construction of the curve $\Gamma$}

Throughout all this section, we assume that the Heisenberg group $\mathbb{H}$ is endowed with its Carnot-Carathéodory metric $d_{c}$. Let $E$ be a compact subset of $\mathbb{H}$. Without loss of generality, we may assume that $\operatorname{diam} E=1$.

For any $j \in \mathbb{N}$, we consider a maximal subset $\Delta_{j}$ of $E$ such that

(i) For any $P \in \Delta_{j}$, any $Q \in \Delta_{j}$ with $P \neq Q, d_{c}(P, Q)>2^{-j}$.

(ii) For any $P \in E$, there exists $Q \in \Delta_{j}$ such that $d_{c}(P, Q) \leq 2^{-j}$.

In other words, $\Delta_{j}$ is a $2^{-j}$-dense subset of $E$ and $\cup_{j \in \mathbb{N}} \Delta_{j}$ is a net associated with $E$. By construction, we may assume that $\Delta_{j} \subset \Delta_{j+1}$ for $j \in \mathbb{N}$. For any $l \in \mathbb{N}$, set $A_{l}=\Delta_{l} \backslash \Delta_{l-1}$. We say that $P \in \cup_{j=0}^{+\infty} \Delta_{j}$ is of generation $l$ if $P \in A_{l}$. Recall that $\left(\mathbb{H}, d_{c}\right)$ is Ahlfors-regular of dimension 4. More precisely, there exists $C_{A R} \geq 1$ such that, for any $P \in \mathbb{H}$, any $r>0$,

$$
\mathcal{H}_{c}^{4}\left(U_{c}(P, r)\right)=C_{A R} r^{4}
$$

Remember $U_{c}(P, r)$ denotes the open ball (with respect to $d_{c}$ ) with center $P$ and radius $r$. Thus, if $P$ is of generation $l$ and if $\delta>0$, the number of points in $A_{l} \cap U_{c}\left(P, \delta 2^{-l}\right)$ is bounded by a constant depending only on $\delta$.

Suppose that $\beta_{\mathbb{H}}(E)<+\infty$. Our goal is to construct a rectifiable curve $\Gamma$ such that $E \subset \Gamma$ and $l(\Gamma) \leq C\left(\mathcal{H}^{1}(E)+\operatorname{diam} E\right)$. Here, $l(\Gamma)$ denotes the length of $\Gamma$ with respect to $d_{c}$. Note that it is enough to construct a continuum $\Gamma$ such that $E \subset \Gamma$ and $\mathcal{H}_{c}^{1}(\Gamma) \leq C\left(\beta_{\mathbb{H}}(E)+\operatorname{diam} E\right)$. To see this, adapt the argument of [9, Theorem 1.8] (see the Appendix). To construct the continuum $\Gamma$, we will follow the algorithm given by P. Jones in [18]. The basic idea is to join the points of $\Delta_{j}$ by geodesics in a reasonnable way to get a continuum $\Gamma_{j}$. The continuum $\Gamma$ will be the limit of the sequence $\left(\Gamma_{j}\right)$. The first key observation is that we need to "order" the points of $\Delta_{j}$ before joining them and we now discuss how to do this. 
Let $F$ be a finite subset of $\mathbb{H}$. We say that $\left[P_{0}, \ldots, P_{n}\right]$ is an order of $F$ if $F=\left\{P_{0}, P_{1}, \ldots, P_{n}\right\}$ and

$$
d_{c}\left(P_{i}, P_{k}\right) \geq \max \left(d_{c}\left(P_{i}, P_{j}\right), d_{c}\left(P_{j}, P_{k}\right)\right)
$$

whenever $i<j<k$. In this case, we say that $F$ is orderable. Note that, if $\left[P_{1}, \ldots, P_{n}\right]$ is an order of $F$, then $\left[P_{n}, \ldots, P_{1}\right]$ is also an order of $F$ (the reverse order). By convention, $[P]$ is an order of $\{P\}$, and $[P, Q]$ (or $[Q, P])$ is an order of $\{P, Q\}$. First, we prove that a subset of an Heisenberg straight line has an order.

Lemma 3.1. Let $F$ be a finite subset of an Heisenberg straight line $L$ in $G(\mathbb{H}, 1)$. Then, $F$ is orderable.

Proof. Without loss of generality, we can assume that $L$ is contained in $\{[x, 0] ; x \in \mathbb{R}\}$. Label the points $P_{0}=\left[x_{0}, 0\right], \ldots, P_{n}=\left[x_{n}, 0\right]$ of $F$ such that $x_{0}<x_{1}<\cdots<x_{n}$. Fix $i<j<k$. Recall that, by Lemma 2.6, $d_{c}\left(P_{i}, P_{k}\right)=$ $d_{c}\left(P_{i}, P_{j}\right)+d_{c}\left(P_{j}, P_{k}\right)$. Thus, $d_{c}\left(P_{i}, P_{k}\right)>\max \left(d_{c}\left(P_{i}, P_{j}\right), d_{c}\left(P_{j}, P_{k}\right)\right)$. Hence, $\left[P_{1}, \ldots, P_{n}\right]$ is an order of $F$.

This result remains true if the set $F$ is sufficiently closed to an Heisenberg straigth line.

Proposition 3.2. Let $E \subset \mathbb{H}$. Fix $C_{0}>c_{0}>0$. Then, there exists $\varepsilon_{0}=$ $c_{0} /\left(100 C_{0}\right)$ such that the following holds. If $P \in \mathbb{H}$ and $t>0$ are such that $\beta_{\mathbb{H}}\left(P, C_{0} t\right) \leq \varepsilon_{0}$ (the $\beta_{\mathbb{H}}$ is associated with the set $\left.E\right)$, and $F \subset E \cap U_{c}\left(P, C_{0} t\right)$ is such that $d_{c}\left(Q, Q^{\prime}\right) \geq c_{0}$ t whenever $Q, Q^{\prime} \in F, Q \neq Q^{\prime}$, then $F$ is finite and orderable.

Proof. The fact that $F$ is finite comes from the Ahlfors-regularity of $\left(\mathbb{H}, d_{c}\right)$. Since $\beta_{\mathbb{H}}\left(P, C_{0} t\right) \leq \varepsilon_{0}$, there exists an Heisenberg straigth line $L$ such that for all $Q \in U_{c}\left(P, C_{0} t\right)$,

$$
d_{c}(Q, L) \leq 2 C_{0} \varepsilon_{0} t
$$

For any $Q \in U_{c}\left(P, C_{0} t\right)$ denote by $\Pi_{L}(Q)$ a point of $L$ such that $d_{c}\left(Q, \Pi_{L}(Q)\right)$ $=d_{c}(Q, L)$. If $Q$ and $Q^{\prime}$ are two distinct points of $F$, then $\Pi_{L}(Q) \neq$ $\Pi_{L}\left(Q^{\prime}\right)$. Indeed, if you assume that $\Pi_{L}(Q)=\Pi_{L}\left(Q^{\prime}\right)$, then $d_{c}\left(Q, Q^{\prime}\right) \leq$ $d_{c}\left(Q, \Pi_{L}(Q)\right)+d_{c}\left(\Pi_{L}(Q), Q^{\prime}\right) \leq 4 C_{0} \varepsilon_{0} t$. Thus, we get a contradiction if $\varepsilon_{0}<c_{0} /\left(4 C_{0}\right)$. By the previous lemma, the set $\Pi_{L}(F):=\left\{\Pi_{L}(Q), Q \in F\right\}$ is orderable. In fact, we can write $\Pi_{L}(F)=\left\{A_{i}, i=1, \ldots, n\right\}$ such that

$$
d_{c}\left(A_{i}, A_{k}\right)=d_{c}\left(A_{i}, A_{j}\right)+d_{c}\left(A_{j}, A_{k}\right)
$$

whenever $i<j<k$. Label now the points $Q_{1}, \ldots, Q_{n}$ of $F$ such that, for any $i=1, \ldots, n, \Pi_{L}\left(Q_{i}\right)=A_{i}$. To conclude, we check that $\left[Q_{1}, \ldots, Q_{n}\right]$ is an order of $F$. 
To see this, fix $i<j<k$. Then, by the triangle inequality and the choice of $L$, we easily get

$$
\begin{aligned}
d_{c}\left(\Pi_{L}\left(Q_{i}\right), \Pi_{L}\left(Q_{k}\right)\right) & \leq d_{c}\left(Q_{i}, Q_{k}\right)+4 \varepsilon_{0} C_{0} t \\
d_{c}\left(Q_{i}, Q_{j}\right) & \leq d_{c}\left(\Pi_{L}\left(Q_{i}\right), \Pi_{L}\left(Q_{j}\right)\right)+4 \varepsilon_{0} C_{0} t, \\
d_{c}\left(Q_{j}, Q_{k}\right) & \leq d_{c}\left(\Pi_{L}\left(Q_{j}\right), \Pi_{L}\left(Q_{k}\right)\right)+4 \varepsilon_{0} C_{0} t .
\end{aligned}
$$

Recall that

$$
\begin{aligned}
d_{c}\left(\Pi_{L}\left(Q_{i}\right), \Pi_{L}\left(Q_{k}\right)\right) & =d_{c}\left(A_{i}, A_{k}\right) \\
& =d_{c}\left(A_{i}, A_{j}\right)+d_{c}\left(A_{j}, A_{k}\right) \\
& =d_{c}\left(\Pi_{L}\left(Q_{i}\right), \Pi_{L}\left(Q_{j}\right)\right)+d_{c}\left(\Pi_{L}\left(Q_{j}\right), \Pi_{L}\left(Q_{k}\right)\right)
\end{aligned}
$$

Hence, we get

$$
d_{c}\left(Q_{i}, Q_{k}\right) \geq d_{c}\left(Q_{i}, Q_{j}\right)+d_{c}\left(Q_{j}, Q_{k}\right)-12 \varepsilon_{0} C_{0} t .
$$

Assume for instance that $\max \left(d_{c}\left(Q_{i}, Q_{j}\right), d_{c}\left(Q_{j}, Q_{k}\right)\right)=d_{c}\left(Q_{i}, Q_{j}\right)$. Since $d_{c}\left(Q_{j}, Q_{k}\right) \geq c_{0} t$, we get $d_{c}\left(Q_{i}, Q_{k}\right)>d_{c}\left(Q_{i}, Q_{j}\right)$ if $\varepsilon_{0}<c_{0} /\left(12 C_{0}\right)$.

We could expect to order a finite set $F \subset \mathbb{H}$ by considering a fixed point $Q$ and labelling the points $P_{1}, \ldots, P_{n}$ of $F$ such that $d_{c}\left(P_{i}, Q\right)$ decreases with $i$. In fact, we have the following result.

Proposition 3.3. Let $F \subset \mathbb{H}$ be a finite set. Let $Q, Q^{\prime}$ in $F$ such that $\operatorname{diam}(F)=d_{c}\left(Q, Q^{\prime}\right)$. Assume that $F$ has an order. Then, if we label the point of $F$ by $P_{1}=Q, P_{2}, \ldots, P_{n}=Q^{\prime}$ such that $d_{c}\left(Q, P_{2}\right)<d_{c}\left(Q, P_{3}\right)<$ $\cdots<d_{c}\left(Q, P_{n}\right)=\operatorname{diamF}$, then $\left[P_{1}, \ldots, P_{n}\right]$ is an order of $F$. More precisely, either $\left[P_{1}, \ldots, P_{n}\right]$ is the given order, or it is the reverse order

Proof. Denote by $\left[A_{1}, \ldots, A_{n}\right]$ an order of $F$. We claim that $A_{i}=P_{i}$ for any $i=1, \ldots, n$ or $A_{i}=P_{n-i}$ for any $i=1, \ldots, n$. Indeed, assume for instance that $P_{1}=A_{i}$ with $i \in\{2, \ldots, n-1\}$ and $P_{n}=A_{j}$ with $i>j$ (the case $i<j$ is the same). Then,

$d_{c}\left(A_{1}, A_{i}\right)=d_{c}\left(A_{1}, Q\right) \geq \max \left(d_{c}\left(A_{1}, A_{j}\right), d_{c}\left(A_{i}, A_{j}\right)\right) \geq d_{c}\left(Q, Q^{\prime}\right)=\operatorname{diam}(F)$.

We get a contradiction. Hence, $\left\{A_{1}, A_{n}\right\}=\left\{P_{1}, P_{n}\right\}$. From now on, assume that $A_{1}=P_{1}$ and $A_{n}=P_{n}$. Assume that $A_{2} \neq P_{2}$. Hence, there exists $i>2$ such that $P_{2}=A_{i}$. This yields to a contradiction:

$$
\left.d_{c}\left(P_{1}, P_{2}\right)=d_{c}\left(A_{1}, A_{i}\right) \geq \max \left(d_{c}\left(A_{1}, A_{2}\right)\right), d_{c}\left(A_{2}, A_{i}\right)\right) \geq d_{c}\left(P_{1}, A_{2}\right) .
$$

Thus, $A_{2}=P_{2}$.

By induction, we easily prove that $A_{i}=P_{i}$ for any $i=2, \ldots, n-1$. Thus, $\left[P_{1}, \ldots, P_{n}\right]$ is an order of $F$. The case $A_{n}=P_{1}$ and $A_{n}=P_{1}$ is similar by noting that $\left[B_{1}, \ldots, B_{n}\right]$ with $B_{i}=A_{n-i}$ is also an order of $F$. 
Remark 3.1. The notion of order could be defined for more general sets in general metric spaces (see [16]): Let $E$ be a subset of a metric space $(X, d)$. Then, $E$ is said to have an order if there exists an injective map $\phi: E \rightarrow \mathbb{R}$ such that, for any distinct points $x, y$ and $z$ in $E$,

$$
\phi(x)<\phi(y)<\phi(z) \Rightarrow d(x, z)>\max (d(x, y), d(y, z)) .
$$

For the simplicity of the presentation, we prefer here to consider finite subsets of the Heisenberg group. However, all the previous results remain true in general Carnot groups.

We now start the construction of the $\Gamma_{j}$ 's. For this, fix $C_{1}, C_{2}>0$ large enough and $\varepsilon_{0}>0$ small enough (see below). On the other hand, the letter $C$ will denote a constant which can vary from one line to the other one. We remind that, if $P$ and $Q$ are in $\mathbb{H},[P, Q]$ denotes a minimizing geodesic segment in $\mathbb{H}$ joining $P$ to $Q$.

To simplify, assume that $\Delta_{0}=\left\{P_{0}, Q_{0}\right\}$. Then, there exist geodesic segments $\left[P_{0}, a\left(P_{0}\right)\right]$ and $\left[Q_{0}, a\left(Q_{0}\right)\right]$ such that $d_{c}\left(P_{0}, a\left(P_{0}\right)\right)=d\left(Q_{0}, a\left(Q_{0}\right)\right)=$ $C_{1} d\left(P_{0}, Q_{0}\right)$. We can always take $a\left(P_{0}\right), a\left(Q_{0}\right)$ such that

$$
d_{c}\left(Q_{0}, a\left(P_{0}\right)\right) \geq C_{1}=C_{1} \operatorname{diam} E, d_{c}\left(P_{0}, a\left(Q_{0}\right)\right) \geq C_{1}=C_{1} \operatorname{diam} E .
$$

This can be proved by contradiction argument thanks to the fact that the $d_{c}$-diameter of $B_{c}(P, r)$ is $2 r$. Then, we set $\Gamma_{0}=\left[a\left(P_{0}\right), P_{0}\right] \cup\left[P_{0}, Q_{0}\right] \cup$ $\left[Q_{0}, a\left(Q_{0}\right)\right]$. Note that

$$
\operatorname{diam} \mathrm{E} \leq l\left(\Gamma_{0}\right) \leq\left(1+4 C_{1}\right) \operatorname{diam} E
$$

Assume by induction that continua $\Gamma_{1}, \ldots, \Gamma_{j-1}$ have been constructed such that, for any $k=1, \ldots, j-1, \Gamma_{k}$ is a connected union of a finite number of geodesic segments, that is $\Gamma_{k}=\bigcup_{P, Q \in \mathcal{G}_{k}}[P, Q]\left(\mathcal{G}_{k}\right.$ is the set of endpoints of geodesics segments of $\Gamma_{k}$ ). Moreover, we assume that the following holds.

(P1) The set $\Delta_{k}$ is contained in the set $\mathcal{G}_{k}$ of endpoints of geodesic segments of $\Gamma_{k}$. Moreover, if $S$ is a geodesic segment in $\Gamma_{k}$, we assume that at least one endpoint of $S$ is in $\Delta_{k}$.

Notations. Let $S$ be a geodesic of $\Gamma_{k}$. We say that $S$ is a primary segment if its two endpoints are in $\Delta_{k}$. Otherwise, we call it a secondary segment (that is, when one endpoint of $S$ is in $\Delta_{k}$, but the other one is outside $\Delta_{k}$ ).

(P2) For any $P \in \Delta_{k}$, if $\Delta_{k} \cap U_{c}\left(P, C_{1} 2^{-k-3}\right)$ has an order $\left[P_{1}, \ldots, P_{n}\right]$, then $\left[P_{i}, P_{i+1}\right] \subset \Gamma_{k}$ (for any $\left.i=1, \ldots, n-1\right)$. 
(P3) For any $P \in \Delta_{k}$, if $\Delta_{k} \cap U_{c}\left(P, C_{1} 2^{-k-3}\right)$ has an order $\left[P_{1}, \ldots, P_{n}\right]$ such that $P=P_{n}$, then $P$ belongs to a segment $[P, \alpha(P)]$ such that $d_{c}(P, \alpha(P)) \geq$ $C_{1} 2^{-k}$ and $\left[P_{n-1}, P, \alpha(P)\right]$ is an order of $\left\{P_{n-1}, P, \alpha(P)\right\}$. Moreover, if $\Delta_{k-1} \cap B\left(P, C_{1} 2^{-k-1}\right)=\{P\}$, then there exists $Q_{1}$ and $Q_{2}$ such that the segments $\left[Q_{1}, P\right]$ and $\left[P, Q_{2}\right]$ are in $\Gamma_{k}$ and have length $\geq C_{1} 2^{-k}$, and $\left[Q_{1}, P, Q_{2}\right]$ is an order of $\left\{Q_{1}, P, Q_{2}\right\}$. To start with let us show that (P1)-(P3) hold at the step $k=0$. As for (P1),(P2), this is trivial. On the other hand, (P3) follows, since $d_{c}\left(a\left(P_{0}\right), Q_{0}\right) \geq C_{1}=\max \left\{d_{c}\left(a\left(P_{0}\right), P_{0}\right), d_{c}\left(P_{0}, Q_{0}\right)\right\}$. Analogously, follows, since $d_{c}\left(a\left(Q_{0}\right), P_{0}\right) \geq C_{1}=\max \left\{d_{c}\left(a\left(Q_{0}\right), Q_{0}\right), d_{c}\left(Q_{0}, P_{0}\right)\right\}$. Finally $\Delta_{k-1}=\emptyset$.

We now start the construction of $\Gamma_{j}$. Let $P \in A_{j}$ (that is $P \in \Delta_{j} \backslash \Delta_{j-1}$ ). Assume that $P \notin \Gamma_{j-1}$ (Otherwise, there is nothing to do). There exists $R_{0} \in \Delta_{j-1}$ such that $d_{c}\left(P, R_{0}\right) \leq 2^{-j+1}$. Note that this implies that $U_{c}\left(R_{0}, C_{1} 2^{-j-2}\right) \subset U_{c}\left(P, C_{1}, 2^{-j}\right)$ (if $C_{1}$ is large enough). By "deforming" $\Gamma_{j-1}$ in the neighborhood of $R_{0}$, we would like to construct a new curve passing through $P$. The fact that the "new" curve $\Gamma_{j}$ satifies $(\mathrm{P} 1)$ and $(\mathrm{P} 3)$ is clear and is left to the reader. We will prove at the end of the construction that $\Gamma_{j}$ satisfies $(\mathrm{P} 2)$.

Case $\mathbf{A}: \beta_{\mathbb{H}}\left(P, C_{1} 2^{-j}\right) \geq \varepsilon_{0}$.

For any $Q \in U_{c}\left(P, C_{1} 2^{-j}\right) \cap \Delta_{j}$, consider a geodesic segment $[Q, a(Q)]$ such that $d_{c}(Q, a(Q))=C_{1} 2^{-j}$ and $[P, Q, a(Q)]$ is an order of $\{P, Q, a(Q)\}$. The "curve" $\Gamma_{j}(S)$ is obtained by adding the geodesic segment $[P, Q]$ and the geodesic segment $[Q, a(Q)]$ for any $Q \in U_{c}\left(P, C_{1} 2^{-j}\right) \cap \Delta_{j}$. Since $\left(\mathbb{H}, d_{c}\right)$ is Ahlfors-regular, $\operatorname{card}\left(U_{c}\left(P, 2^{-j}\right) \cap \Delta_{j}\right)$ is bounded uniformly in $j$. Hence,

$$
l\left(\Gamma_{j}\right)-l\left(\Gamma_{j-1}\right) \leq C 2^{-j} \leq \frac{C}{\varepsilon_{0}^{2}} 2^{-j} \beta_{\mathbb{H}}\left(P, C_{1} 2^{-j}\right)^{2} \leq C \beta_{\mathbb{H}}\left(P, C_{1} 2^{-j}\right)^{2} 2^{-j}
$$

We notice that the symbol $\Gamma_{j}$ is not correct since what we are dealing with is more precisely the localization of $\Gamma_{j}$ around the point $P$ at the scale $2^{-j}$. Hence at the very end we have to sum up over all the localizations. Nevertheless we proceed in this way to avoid cumbersome notations.

Case B: $\beta_{\mathbb{H}}\left(P, C_{1} 2^{-j}\right) \leq \varepsilon_{0}$.

In this case, by proposition $3.2, \Delta_{j} \cap U_{c}\left(P, C_{1} 2^{-j}\right)$ has (at least) one order $\left[P_{1}, \ldots, P_{n}\right]$. This order implies an order on the set $\Delta_{j-1} \cap U_{c}\left(P, C_{1} 2^{-j}\right)$ (and also on $\Delta_{j-1} \cap U_{c}\left(R_{0}, C_{1} 2^{-j-2}\right)$ ) in a natural way. Indeed, if we denote by $m$ the cardinal of $\Delta_{j-1} \cap U_{c}\left(P, C_{1} 2^{-j}\right)$, we can consider an increasing map $r:\{1, \ldots, m\} \rightarrow\{1, \ldots, n\}$ such that $\left[P_{r(1)}, \ldots, P_{r(m)}\right]$ is an order of $\Delta_{j-1} \cap U_{c}\left(P, C_{1} 2^{-j}\right)$. We emphasize on the fact that property (P2) will be maintained at this step of the construction since we will join the points of $\Delta_{j} \cap U_{c}\left(P, C_{1} 2^{-j}\right)$ by following the order $\left[P_{1}, \ldots, P_{n}\right]$. 
Case B1: There exists an order $\left[Q_{1}, \ldots, Q_{m}\right]$ of $\Delta_{j-1} \cap U_{c}\left(P, C_{1} 2^{-j}\right)$ such that $R_{0} \neq Q_{1}$ and $R_{0} \neq Q_{m}$. Hence, there exists $i_{0} \in\{2, \ldots, m-1\}$ such that $R_{0}=Q_{i_{0}}$. Then, there exists an order $\left[P_{1}, \ldots, P_{n}\right]$ of $\Delta_{j} \cap U_{c}\left(P, C_{1} 2^{-j}\right)$ and an increasing map $r:\{1, \ldots, m\} \rightarrow\{1, \ldots, n\}$ such that $Q_{i}=P_{r(i)}$. Recall that by property (P2), $\left[Q_{i_{0}-1}, Q_{i_{0}}\right]$ and $\left[Q_{i_{0}}, Q_{i_{0}+1}\right]$ are in $\Gamma_{j-1}$.

Then, $\Gamma_{j}$ is obtained by replacing $\left[Q_{i_{0}-1}, Q_{i_{0}}\right] \cup\left[Q_{i_{0}}, Q_{i_{0}+1}\right]$ by $\left[P_{r\left(i_{0}-1\right)}, P_{r\left(i_{0}-1\right)+1}\right] \cup \ldots\left[P_{r\left(i_{0}\right)-1}, P_{r\left(i_{0}\right)}\right] \cup\left[P_{r\left(i_{0}\right)}, P_{r\left(i_{0}\right)+1}\right] \cdots \cup\left[P_{r\left(i_{0}+1\right)-1}, P_{r\left(i_{0}+1\right)}\right]$.

We should now estimate

$$
\begin{aligned}
l\left(\Gamma_{j}\right)-l\left(\Gamma_{j-1}\right) & =\sum_{\substack{k=r\left(i_{0}-1\right)\\
}}^{r\left(i_{0}+1\right)-1} d_{c}\left(P_{k}, P_{k+1}\right)-\left(d_{c}\left(Q_{i_{0}-1}, Q_{i_{0}}\right)+d_{c}\left(Q_{i_{0}}, Q_{i_{0}+1}\right)\right) \\
&
\end{aligned}
$$

where

$$
\begin{aligned}
& \mathcal{S}_{1}=\sum_{k=r\left(i_{0}-1\right)}^{r\left(i_{0}\right)-1} d_{c}\left(P_{k}, P_{k+1}\right)-d_{c}\left(P_{r\left(i_{0}-1\right)}, P_{r\left(i_{0}\right)}\right), \\
& \mathcal{S}_{2}=\sum_{k=r\left(i_{0}\right)}^{r\left(i_{0}+1\right)-1} d_{c}\left(P_{k}, P_{k+1}\right)-d_{c}\left(P_{r\left(i_{0}\right)}, P_{r\left(i_{0}+1\right)}\right) .
\end{aligned}
$$

Now we estimate $\mathcal{S}_{1}$. Note that, if $r\left(i_{0}-1\right)=r\left(i_{0}\right)-1$, then $\mathcal{S}_{1}=0$. This happens when there is no points of $A_{j}$ "between" $Q_{i_{0}-1}$ and $Q_{i_{0}}$. From now on, we assume that we are not in this situation. To simplify the notation, we assume that $r\left(i_{0}-1\right)=1$ and $r\left(i_{0}\right)=s_{0}$. Then,

$$
\begin{aligned}
\mathcal{S}_{1} & =\sum_{k=1}^{s_{0}-1} d_{c}\left(P_{k}, P_{k+1}\right)-d_{c}\left(P_{1}, P_{s_{0}}\right) \\
& =\sum_{k=1}^{s_{0}-2}\left(d_{c}\left(P_{k}, P_{k+1}\right)+d_{c}\left(P_{k+1}, P_{s_{0}}\right)-d_{c}\left(P_{k}, P_{s_{0}}\right)\right) .
\end{aligned}
$$

We now should estimate $d_{c}\left(P_{k}, P_{k+1}\right)+d_{c}\left(P_{k+1}, P_{s_{0}}\right)-d_{c}\left(P_{k}, P_{s_{0}}\right)$ for any $k=1, \ldots, s_{0}-2$. To do this, we apply corollary 2.16. Since $k<k+1<s_{0}$, the fact that $\left[P_{1}, P_{2}, \ldots, P_{n}\right]$ is an order gives

$$
d_{c}\left(P_{k}, P_{s_{0}}\right) \geq \max \left(d_{c}\left(P_{k}, P_{k+1}\right), d_{c}\left(P_{k+1}, P_{s_{0}}\right) .\right.
$$

Moreover, the distances $d_{c}\left(P_{k}, P_{s_{0}}\right), d_{c}\left(P_{k}, P_{k+1}\right)$ and $d_{c}\left(P_{k+1}, P_{s_{0}}\right)$ are mutually comparable. Thus, if $\varepsilon_{0}$ is small enough, by corollary 2.16, we get

$$
d_{c}\left(P_{k}, P_{k+1}\right)+d_{c}\left(P_{k+1}, P_{s_{0}}\right)-d_{c}\left(P_{k}, P_{s_{0}}\right) \leq C \beta_{\mathbb{H}}\left(P, C_{1} 2^{-j}\right)^{2} 2^{-j}
$$

Since the cardinal of $U_{c}\left(P, C_{1} 2^{-j}\right) \cap \Delta_{j}$ is uniformly bounded, we get

$$
\mathcal{S}_{1} \leq C \beta_{\mathbb{H}}\left(P, C_{1} 2^{-j}\right)^{2} 2^{-j}
$$


For the same reason, we get the same estimate for $\mathcal{S}_{2}$. Eventually, this yields to

$$
l\left(\Gamma_{j}\right)-l\left(\Gamma_{j-1}\right) \leq C \beta_{\mathbb{H}}\left(P, C_{1} 2^{-j}\right)^{2} 2^{-j}
$$

Case B2: There exists an order $\left[S_{1}, \ldots, S_{m}\right]$ of $\Delta_{j-1} \cap U_{c}\left(P, C_{1} 2^{-j}\right)$ such that $R_{0}=S_{1}$ or $R_{0}=S_{m}$. Thus, by property (P3), there exists (at least) one segment $\gamma_{R_{0}}=\left[R_{0}, \alpha\left(R_{0}\right)\right]$ such that $l\left(\gamma_{R_{0}}\right) \geq C_{1} 2^{-j+1} \geq C_{1} 2^{-j}$. This segment is either a primary segment or a secondary segment. A crucial observation is that this segment belongs to the continuum $\Gamma$ (which is obtained as a limit of the $\Gamma_{j}$ 's). In fact, for any $k \geq j,\left[R_{0}, \alpha\left(R_{0}\right)\right] \subset \Gamma_{k}$. Indeed, a segment of some $\Gamma_{k}$ could be modified only by the method described in case B1 (see for instance what happens to the segment $\left[Q_{i_{0}-1}, Q_{i_{0}}\right]$ in case B1 above). But, this method is used to modify primary segments of length less than $C_{1} 2^{-k}$. This is not the case for $\left[R_{0}, \alpha\left(R_{0}\right)\right]$ for any $k \geq j$.

Notation: Let $\mathcal{A}$ be the set of (geodesic) segments $\left[R, R^{\prime}\right]$ such that there exists $k \in \mathbb{N}$ so that for, any $l \geq k, R, R^{\prime} \in \mathcal{G}_{l}$ (that is $\left[R, R^{\prime}\right] \subset \Gamma_{l}$ ). For $I \in \mathcal{A}$, we denote by $\tau_{1}(I)$ (respectively $\tau_{2}(I)$ ) the total length added to $I$ by applying case 1 (respectively case 2 ) of case B2(i).

Case B2(i): $U_{c}\left(P, C_{1} 2^{-j}\right) \cap \Delta_{j-1}=\left\{R_{0}\right\}$.

Then, by property (P3), there exist $Q_{1}$ and $Q_{2}$ such that the segments $\left[Q_{1}, R_{0}\right]$ and $\left[R_{0}, Q_{2}\right]$ are in $\Gamma_{j-1}$ and have length $\geq C_{1} 2^{-j}$. Moreover, $\left[Q_{1}, R_{0}, Q_{2}\right]$ is an order of $\left\{Q_{1}, R_{0}, Q_{2}\right\}$, that is,

$$
d_{c}\left(Q_{1}, Q_{2}\right) \geq \max \left(d_{c}\left(Q_{1}, R_{0}\right), d_{c}\left(R_{0}, Q_{2}\right)\right) .
$$

We would like to insert points "between" $Q_{1}$ and $R_{0}$ (the case of points between $R_{0}$ and $Q_{2}$ is similar).

Case 1. $d_{c}\left(Q_{1}, R_{0}\right) \geq C_{2} 2^{-j}$.

By proposition 3.2, there exists an order $\left[P_{1}, \ldots, P_{n}\right]$ of $U_{c}\left(P, C_{1} 2^{-j}\right) \cap \Delta_{j}$. We first consider the points $Q_{1}, P_{1}, \ldots, P_{i_{0}}$ where $R_{0}=P_{i_{0}}$. We assume that such a point $P_{1}$ exists (Otherwise, there is nothing to do).

Consider a point $a\left(P_{1}\right)$ such that $d_{c}\left(a\left(P_{1}\right), P_{1}\right)=C_{1} 2^{-j}$ and $\left[a\left(P_{1}\right), P_{1}, R_{0}\right]$ is an order of $\left\{a\left(P_{1}\right), P_{1}, R_{0}\right\}$. Then, $\Gamma_{j}$ is obtained by adding to $\Gamma_{j-1}$ the segments $\left[a\left(P_{1}\right), P_{1}\right] \cup\left[P_{1}, P_{2}\right] \cup \cdots \cup\left[P_{i_{0}-1}, P_{i_{0}}\right]$. Since the cardinal of $U_{c}\left(P, C_{1} 2^{-j}\right) \cap \Delta_{j}$ is bounded (uniformly in $P$ and $j$ ), we get

$$
\begin{aligned}
l\left(\Gamma_{j}\right)-l\left(\Gamma_{j-1}\right) & \left.=d_{c}\left(a\left(P_{1}\right), P_{1}\right)+\sum_{k=1}^{i_{0}-1} d_{c}\left(P_{k}, P_{k+1}\right)\right) \\
& \leq\left(C_{1}+C\right) 2^{-j} .
\end{aligned}
$$


It could happen that the same method involving the segment $\left[Q_{1}, R_{0}\right]$ has been used to construct $\Gamma_{k}(k<j)$ or will be used to construct $\Gamma_{k}(k>j)$. Denote by $l_{0}\left(l_{0} \leq j\right)$ the first generation for which this happens. Note that $d_{c}\left(Q_{1}, R_{0}\right) \geq C_{2} 2^{-l_{0}}$. Hence, since the same situation could happen at $Q_{1}$, we get

$$
\begin{aligned}
\tau_{1}\left(\left[Q_{1}, R_{0}\right]\right) & \leq 2 \sum_{k=l_{0}}^{+\infty}\left(C_{1}+C\right) 2^{-j} \\
& \leq 4\left(C+C_{1}\right) 2^{-l_{0}} \\
& \leq 4 \frac{C+C_{1}}{C_{2}} d_{c}\left(Q_{1}, R_{0}\right) .
\end{aligned}
$$

Thus, if $C_{2}$ is big enough, we get

$$
\tau_{1}\left(\left[Q_{1}, R_{0}\right]\right) \leq \frac{d_{c}\left(Q_{1}, R_{0}\right)}{10^{6}}
$$

We use the same method for $P_{i_{i_{0}}}, \ldots, P_{n}, Q_{2}\left(\right.$ if $\left.d_{c}\left(R_{0}, Q_{2}\right) \geq C_{2} 2^{-j}\right)$ and we get the same estimate, that is

$$
\tau_{1}\left(\left[Q_{2}, R_{0}\right]\right) \leq \frac{d_{c}\left(Q_{2}, R_{0}\right)}{10^{6}} .
$$

Case 2. $d_{c}\left(Q_{1}, R_{0}\right) \leq C_{2} 2^{-j}$.

Fix $N_{0} \in \mathbb{N}$ big enough. We go directly to the step $j+N_{0}$ of the construction. By proposition 3.2, there exists an order $\left[P_{0}=Q_{1}, P_{1}, \ldots, P_{m}\right]$ of $\left(U_{c}\left(P, 10.2^{-j}\right) \cap \Delta_{j+N_{0}}\right) \cup\left\{Q_{1}\right\}$. Then, there exists $n \in\{1, \ldots, m\}$ such that $P_{n}=R_{0}$. We first consider the points $P_{0}, P_{1}, \ldots, P_{n}$. Choose a point $a\left(P_{1}\right)$ such that $d_{c}\left(P_{1}, a\left(P_{1}\right)\right)=C_{1} 2^{-j-N_{0}}$ and $\left[a\left(P_{1}\right), P_{1}, R_{0}\right]$ is an order of $\left\{a\left(P_{1}\right), P_{1}, R_{0}\right\}$. First, we add to $\Gamma_{j-1}$ the segments $\left[a\left(P_{1}\right), P_{1}\right] \cup\left[P_{1}, R_{0}\right]$. Then, $\Gamma_{j+N_{0}}$ is obtained by replacing $\left[P_{1}, R_{0}\right]$ by $\left[P_{1}, P_{2}\right] \cup \cdots \cup\left[P_{n-1}, R_{0}\right]$. The key observation is that this case is used only one time for the segment $\left[Q_{1}, R_{0}\right]$ at $R_{0}$. Indeed, the next time this situation will occur at $R_{0}$, we will use the segment $\left[P_{n-1}, R_{0}\right]$ instead of the segment $\left[Q_{1}, R_{0}\right]$.

Thus, since the same situation could also happen at $Q_{1}$, we get

$\left.\tau_{2}\left(\left[R_{0}, Q_{1}\right]\right) \leq 2\left(d_{c}\left(a\left(P_{1}\right), P_{1}\right)\right)+d_{c}\left(P_{1}, R_{0}\right)\right)+2\left(\sum_{k=1}^{n-1} d_{c}\left(P_{k}, P_{k+1}\right)-d_{c}\left(P_{1}, R_{0}\right)\right)$.

By using the same method as in case B1, we get that

$$
\sum_{k=1}^{n-1} d_{c}\left(P_{k}, P_{k+1}\right)-d_{c}\left(P_{1}, R_{0}\right) \leq C \beta_{\mathbb{H}}\left(P, C_{1} 2^{-j}\right)^{2} 2^{-j}
$$


Moreover, if $C_{1}$ is big enough, we have

$\left.d_{c}\left(a\left(P_{1}\right), P_{1}\right)\right)+d_{c}\left(P_{1}, P\right) \leq C_{1} 2^{-j-N_{0}}+10.2^{-j} \leq \frac{C_{1}}{10^{3}} 2^{-j} \leq \frac{1}{10^{3}} d_{c}\left(Q_{1}, R_{0}\right)$.

Hence,

$$
\tau_{2}\left(\left[Q_{1}, P\right]\right) \leq C \beta_{\mathbb{H}}\left(P, C_{1} 2^{-j}\right)^{2} 2^{-j}+\frac{1}{10^{3}} d_{c}\left(Q_{1}, R_{0}\right)
$$

We use the same method for $P_{n}=R_{0}, \ldots, P_{m}, Q_{2}\left(\right.$ if $\left.d_{c}\left(R_{0}, Q_{2}\right) \leq C_{2} 2^{-j}\right)$ and we get the same estimate, that is

$$
\tau_{2}\left(\left[Q_{2}, R_{0}\right]\right) \leq C \beta_{\mathbb{H}}\left(P, C_{1} 2^{-j}\right)^{2} 2^{-j}+\frac{1}{10^{3}} d_{c}\left(Q_{1}, P\right) .
$$

Case B2 (ii): $U_{c}\left(P, C_{1} 2^{-j}\right) \cap \Delta_{j-1} \neq\left\{R_{0}\right\}$. Thus, there exists an order $\left[S_{1}, \ldots, S_{m}\right]$ of $\Delta_{j-1} \cap U_{c}\left(P, C_{1} 2^{-j}\right)$ such that $R_{0}=S_{m}$ and $m>1$. By proposition 3.2, there exists an order $\left[P_{1}, \ldots, P_{n}\right]$ of $U_{c}\left(P, C_{1} 2^{-j}\right) \cap \Delta_{j}$ such that there exist $j_{0}<i_{0}$ with $S_{m-1}=P_{j_{0}}$ and $R_{0}=P_{i_{0}}$. For the points $P_{j_{0}}, P_{j_{0}+1}, \ldots, P_{i_{0}}$, use case B1. For the points $P_{i_{0}}, P_{i_{0}+1}, \ldots, P_{n}$, use case B2 (i).

The continuum $\Gamma_{j}$ is constructed by applying the previous procedure to any point $P \in \Delta_{j}$. We now check that $\Gamma_{j}$ satisfies (P2). For this, consider $P \in \Delta_{j}$ such that $U_{c}\left(P, C_{1} 2^{-j-2}\right) \cap \Delta_{j}$ has an order $\left[P_{1}, \ldots, P_{n}\right]$. Fix $i \in\{1, \ldots, n-1\}$.

Case 1. $P_{i}$ or $P_{i+1}$ belongs to $A_{j}=\Delta_{j} \backslash \Delta_{j-1}$. Assume for instance that $P_{i} \in A_{j}$. If $\beta_{\mathbb{H}}\left(P_{i}, C_{1} 2^{-j}\right) \leq \varepsilon_{0}$, then $P_{i}$ has been inserted in $\Gamma_{j}$ by following the order in $U_{c}\left(P_{i}, C_{1} 2^{-j}\right) \cap \Delta_{j}$. Hence, $\left[P_{i}, P_{i+1}\right] \subset \Gamma_{j}$. If $\beta_{\mathbb{H}}\left(P_{i}, C_{1} 2^{-j}\right) \geq \varepsilon_{0}$, $\Gamma_{j}$ has been obtained by joining $P_{i}$ to any points of $U_{c}\left(P_{i}, C_{1} 2^{-j}\right) \cap \Delta_{j}$. Hence, $\left[P_{i}, P_{i+1}\right] \subset \Gamma_{j}$.

Case 2. $P_{i}$ and $P_{i+1}$ are not in $A_{j}$. Denote by $l_{i}$ and $l_{i+1}$ the integers such that $P_{i} \in A_{l_{i}}$ and $P_{i+1} \in A_{l_{i+1}}$. Assume for instance that $l_{i} \geq l_{i+1}$. Note that $P_{i}$ and $P_{i+1}$ are also neighbour in $\Delta_{l_{i}}$. If there exists an order in $U_{c}\left(P_{i}, C_{1} 2^{-l_{i}}\right) \cap \Delta_{l_{i}}$ (that is $\left.\beta_{\mathbb{H}}\left(P_{i}, C_{1} 2^{-l_{i}}\right) \leq \varepsilon_{0}\right)$, then since $\Gamma_{l_{i}}$ satisfies (P2) by induction, $\left[P_{i}, P_{i+1}\right] \subset \Gamma_{l_{i}}$. Hence, since $P_{i}$ and $P_{i+1}$ are also neighbour in $\Delta_{j}$, it follows that case $B$ has never been used to insert points between $P_{i}$ and $P_{i+1}$ in the step $k=l_{i}, \ldots, j$ of the construction. Thus, $\left[P_{i}, P_{i+1}\right] \subset \Gamma_{j}$. If $\beta_{\mathbb{H}}\left(P_{i}, C_{1} 2^{-j}\right) \geq \varepsilon_{0}$, we conclude as in case 1 .

By iterating the following algorithm, we construct a sequence of continua $\left(\Gamma_{j}\right)$ such that, for any $j \in \mathbb{N}, \Delta_{j} \subset \Gamma_{j}$. By theorem 5.1 (see the appendix), $\left(\Gamma_{j}\right)$ converges (up to a subsequence) to a continuum $\Gamma$ which contains $E$. 
Moreover, by (3.3), (3.4), (3.10), (3.11), we get

$$
\begin{aligned}
H^{1}(\Gamma) & \leq \sum_{j \in \mathbb{N}}\left(l\left(\Gamma_{j+1}\right)-l\left(\Gamma_{j}\right)\right)+l\left(\Gamma_{0}\right) \\
& \leq C \sum_{j \in \mathbb{N}} \sum_{P \in \Delta_{j}} \beta_{\mathbb{H}}\left(P, C_{3} 2^{-j}\right)^{2} 2^{-j}+\sum_{I \in \mathcal{A}} \tau_{1}(I)+\sum_{I \in \mathcal{A}} \tau_{2}(I)+C \operatorname{diam} E \\
& \leq C \sum_{j \in \mathbb{N}} \sum_{P \in \Delta_{j}} \beta_{\mathbb{H}}\left(P, C_{3} 2^{-j}\right)^{2} 2^{-j}+\frac{1}{10^{6}} H^{1}(\Gamma)+\frac{1}{10^{3}} H^{1}(\Gamma)+C \operatorname{diam} E .
\end{aligned}
$$

Recall that the first term of the previous sum comes from case A and case B1, the second and the third one come from case B2, and the last one is the length of $\Gamma_{0}$. Note that $\sum_{j \in \mathbb{N}} \sum_{P \in \Delta_{j}} \beta_{\mathbb{H}}\left(P, C_{3} 2^{-j}\right)^{2} 2^{-j}$ is a discrete version of $\beta_{H}(E)$. Hence, $H^{1}(\Gamma) \leq C\left(\beta_{\mathbb{H}}(E)+\operatorname{diam} E\right)$ and the proof of the main theorem is complete.

\section{Subsets of regular curves}

In this section, we prove Theorem 1.4. A simple curve $\gamma:[0, T] \rightarrow \mathbb{H}$ is a $\mathbf{C}^{1, \alpha}$-regular horizontal Jordan (i.e. injective) curve if $\gamma=\left(\gamma_{1}, \gamma_{2}, \gamma_{3}\right)$ with

1. $\gamma$ is injective and continuously differentiable, with $\gamma^{\prime}(t) \neq 0$ for $t \in$ $[0, T]$

2. $\left|\gamma^{\prime}(t)-\gamma^{\prime}(s)\right| \leq c|t-s|^{\alpha}$ for all $t, s \in[0, T]$;

3. $\gamma_{3}^{\prime} \equiv 2\left(\gamma_{1}^{\prime} \gamma_{2}-\gamma_{1} \gamma_{2}^{\prime}\right)$.

Proof of Theorem 1.4. Put $m:=\min _{0 \leq s \leq T}\left|\gamma^{\prime}(s)\right|>0$. If $\Omega$ is a bounded fixed neighborhood of $\gamma$ and $t, \theta \in[0, T]$, then, by Proposition 2.4 and (2.3),

$$
\begin{aligned}
& d_{c}(\gamma(\theta), \gamma(t)) \geq \alpha_{1} d(\gamma(\theta), \gamma(t)) \\
& \quad \geq \alpha_{1} c_{1}(\Omega)|\gamma(\theta)-\gamma(t)|=\alpha_{1} c_{1}(\Omega)\left|\int_{0}^{\theta-t} \gamma^{\prime}(t+s) d s\right| \\
& \quad=\alpha_{1} c_{1}(\Omega)\left|\int_{0}^{\theta-t}\left(\gamma^{\prime}(t+s)-\gamma^{\prime}(t)\right) d s+(\theta-t) \gamma^{\prime}(t)\right| \\
& \quad \geq m \alpha_{1} c_{1}(\Omega)|\theta-t|-c \int_{0}^{|\theta-t|}|s|^{\alpha} d s \geq \frac{m}{2} \alpha_{1} c_{1}(\Omega)|\theta-t|,
\end{aligned}
$$

provided $|\theta-t| \leq \delta_{0}, \delta_{0}$ depending only on $\gamma$. We want to show now that there exists $\epsilon_{0}>0$ such that

$$
d_{c}(\gamma(\theta), \gamma(t)) \geq \frac{m}{4} \alpha_{1} c_{1}(\Omega)|\theta-t|:=\frac{1}{c_{2}}|\theta-t|
$$

for any $t, \theta \in[0, T]$ such that $d_{c}(\gamma(\theta), \gamma(t))<\epsilon_{0}$. 
By contradiction, assume there exist $t_{n}, \theta_{n} \in[0, T]$ such that

$$
d_{c}\left(\gamma\left(\theta_{n}\right), \gamma\left(t_{n}\right)\right) \rightarrow 0 \text { as } n \rightarrow \infty
$$

and

$$
d_{c}\left(\gamma\left(\theta_{n}\right), \gamma\left(t_{n}\right)\right)<\frac{m}{4} \alpha_{1} c_{1}(\Omega)\left|\theta_{n}-t_{n}\right| .
$$

By compactness, we can assume that $\theta_{n} \rightarrow \theta$ and $t_{n} \rightarrow t$ as $n \rightarrow \infty$. Hence $\gamma(\theta)=\gamma(t)$ that yields $t=\theta$, since $\gamma$ is simple. Thus $\left|\theta_{n}-t_{n}\right|<\delta_{0}$ for $n$ large, and (4.3) contradicts (4.1). Hence (4.2) is proved.

In addition, before attacking the core of the proof, we notice that our assumptions on $\gamma$ are invariant under group translations, in the sense that, if $q \in \gamma$ and we denote by $\gamma_{q}$ the curve $\tau_{q^{-1}}(\gamma)$, then $\gamma_{q}$ still satisfies (1), (2) and (3) with constants that can be chosen independently of $q \in \gamma$. This properties relies on the fact that the map $(p, q) \rightarrow \tau_{q^{-1}}(p)$ can be written as a polynomial of degree 2 in $p$ and $q$ (of degree 1 in $p$ for fixed $q$ ), so that

$$
\left|\frac{\partial \tau_{q^{-1}}}{\partial p}\right| \leq C \text { for } p \in \mathbb{H} \text { and } q \in \gamma \text {. }
$$

Let now $t \in[0, T]$ be fixed, and let $p_{0}$ be such that $d_{c}\left(p_{0}, \gamma\right)<t$. Then there exists $q_{0} \in \gamma \cap U_{c}(p, t)$. If $r \in G(\mathbb{H}, 1)$ is arbitrary, then

$$
\sup _{p \in \gamma \cap U_{c}\left(p_{0}, t\right)} \frac{d_{c}(p, r)}{t} \leq \sup _{p \in \gamma \cap U_{c}\left(q_{0}, 3 t\right)} \frac{d_{c}(p, r)}{t},
$$

so that

$$
\beta_{\mathbb{H}}\left(p_{0}, t\right) \leq \sup _{p \in \gamma \cap U_{c}\left(q_{0}, 3 t\right)} \frac{d_{c}(p, r)}{t}
$$

for any $r \in G(\mathbb{H}, 1)$. In particular, (4.4) holds if $r$ is the tangent straight line to $\gamma$ at $q_{0}$ (notice since we are dealing with an horizontal curve, then its Euclidean tangent straight line is a lateral of a 1-dimensional subgroup of $\mathbb{H}$ - see e.g. [12]).

Keeping in mind the invariance of the distance $d_{c}$ under group translations and rotations around the $t$-axis, as well as our previous remark on the invariance of our assumptions, we can assume without loss of generality that $q_{0}=0$ and $r=\{(\rho, 0,0), \rho \in \mathbb{R}\}$. Then, if $p \in \gamma \cap U_{c}\left(q_{0}, 3 t\right)$, we can write $p=\gamma(\theta), 0 \leq \theta \leq T$, and we have

$$
\begin{aligned}
d_{c}(p, r)^{4} & \leq c_{3} \inf _{s \in \mathbb{R}}\left\{\left(\left(\gamma_{1}(\theta)-s\right)^{2}+\gamma_{2}^{2}(\theta)\right)^{2}+\left(\gamma_{3}(\theta)-2 s \gamma_{2}(\theta)\right)^{2}\right\} \\
& \leq c_{3}\left\{\gamma_{2}^{4}(\theta)+\left(\gamma_{3}(\theta)-2 \gamma_{1}(\theta) \gamma_{2}(\theta)\right)^{2}\right\} .
\end{aligned}
$$

Keeping in mind that $\gamma_{2}(0)=\gamma_{2}^{\prime}(0)=0$, for a suitable $\sigma \in(0,1)$ we have:

$$
\left|\gamma_{2}(\theta)\right|=\left|\gamma_{2}(\theta)-\gamma_{2}(0)\right|=|\theta|\left|\gamma_{2}^{\prime}(\sigma \theta)-\gamma_{2}^{\prime}(0)\right| \leq C|\theta|^{1+\alpha}
$$


Now, if $d_{c}\left(\gamma_{2}(\theta), 0\right)<\epsilon_{0}$ then $|\theta| \leq c_{2} d_{c}(\gamma(\theta), 0)$. But $d_{c}(\gamma(\theta), 0)=$ $d_{c}(p, 0)<3 t$, so that the assumption $d_{c}\left(\gamma_{2}(\theta), 0\right)<\epsilon_{0}$ is satisfied provided $t$ is small enough $\left(3 c_{2} t<\epsilon_{0}\right)$. Hence, if $t$ is small enough,

$$
\left|\gamma_{2}(\theta)\right| \leq c_{4} t^{1+\alpha}
$$

On the other hand

$$
\gamma_{3}(\theta)-2 \gamma_{1}(\theta) \gamma_{2}(\theta)=\theta\left(\gamma_{3}^{\prime}(\sigma)-2 \gamma_{1}^{\prime}(\sigma) \gamma_{2}(\sigma)-2 \gamma_{1}(\sigma) \gamma_{2}^{\prime}(\sigma)\right)
$$

with $|\sigma|<|\theta|$. But

$$
\gamma_{3}^{\prime}=2\left(\gamma_{1}^{\prime} \gamma_{2}-\gamma_{1} \gamma_{2}^{\prime}\right)
$$

so that

$$
\left|\gamma_{3}(\theta)-2 \gamma_{1}(\theta) \gamma_{2}(\theta)\right|=4|\theta|\left|\gamma_{1}(\sigma) \gamma_{2}^{\prime}(\sigma)\right| \leq C|\theta||\sigma|^{1+\alpha}
$$

since $\gamma_{1}(0)=0, \gamma_{2}^{\prime}(0)=0$. On the other hand

$$
|\sigma|^{1+\alpha} \leq|\theta|^{1+\alpha} \leq c_{5} t^{1+\alpha}
$$

so that

$$
\left|\gamma_{3}(\theta)-2 \gamma_{1}(\theta) \gamma_{2}(\theta)\right| \leq c_{6} t^{2+\alpha}
$$

This yields

$$
d_{c}(p, r) \leq c_{7} t^{1+\frac{\alpha}{2}}
$$

Replacing in (4.4), we get

$$
\beta_{\mathbb{H}}\left(p_{0}, t\right) \leq c_{8} t^{\alpha / 2}
$$

provided $t \leq \epsilon_{0}$. On the other hand

$$
d_{c}(p, r) \leq d_{c}\left(p, q_{0}\right) \leq 3 t
$$

and hence, if $\epsilon_{0} \leq t \leq 1$,

$$
\beta_{\mathbb{H}}\left(p_{0}, t\right) \leq \frac{3}{\epsilon_{0}^{\alpha / 2}} t^{\alpha / 2}
$$

so that (4.5) holds for $0<t \leq 1$.

Eventually we have

$$
\int_{\mathbb{H}} d p \int_{0}^{1} \frac{d t}{t^{4}} \beta_{\mathbb{H}}^{2}(p, t) d t \leq c_{9} \int_{0}^{1} \frac{d t}{t^{4}}\left|\left\{q \in \mathbb{H}: \quad d_{c}(p, \gamma)<t\right\}\right| t^{\alpha} d t
$$


Let us show that

$$
\left|\left\{q \in \mathbb{H}: d_{c}(p, \gamma)<t\right\}\right| \leq c_{10} \ell(\gamma) t^{3},
$$

where $\ell(\gamma)$ is the length of $\gamma$ with respect to Carnot-Carathéodory distance. By definition

$$
\left\{q \in \mathbb{H}: d_{c}(p, \gamma)<t\right\} \subset \bigcup_{p \in \gamma} U_{c}(p, t) .
$$

By Vitali covering lemma, there exists a (finite, by compactness) family of points $\left\{p_{j}: j \in J\right\}$ such that

i) $\left\{q \in \mathbb{H}: d_{c}(p, \gamma)<t\right\} \subset \bigcup_{j \in J} U_{c}\left(p_{j}, 5 t\right)$.

ii) $U_{c}\left(p_{i}, t\right) \cap U_{c}\left(p_{j}, t\right)=\emptyset, \quad i \neq j$.

Now ii) implies that

$$
\sum_{j \in J} \ell\left(\gamma \cap U_{c}\left(p_{j}, t\right)\right) \leq \ell(\gamma)
$$

Consider now $j$ fixed and notice that there exists at least a point $q \in \gamma$ such that $q \notin U_{c}\left(p_{j}, t\right)$, so that if $p_{j}=\gamma\left(\theta_{j}\right)$, we can take $\tilde{p}_{j}$ of the form $\tilde{p}_{j}=\gamma\left(\tilde{\theta}_{j}\right)$, where either

$$
\tilde{\theta}_{j}=\sup \left\{\theta>\theta_{j}: d_{c}\left(\gamma(s), p_{j}\right)<r, \text { for } 0<s<\theta_{j}\right\}
$$

or

$$
\tilde{\theta}_{j}=\inf \left\{\theta<\theta_{j}: d_{c}\left(\gamma(s), p_{j}\right)<r, \text { for } 0<s<\theta_{j}\right\}
$$

In other words, $\tilde{p}_{j}$ is the first point where $\gamma$ reaches the boundary of $U_{c}\left(p_{j}, t\right)$. Denoting by $\gamma_{j}$ the arc of $\gamma$ between $p_{j}$ and $\gamma\left(\tilde{\theta}_{j}\right)$,

$$
t=d_{c}\left(\gamma\left(\tilde{\theta}_{j}\right), p_{j}\right) \leq \ell\left(\gamma_{j}\right) \leq \ell\left(\gamma \cap U_{c}\left(p_{j}, t\right)\right) .
$$

Replacing in (4.8) we have $\operatorname{card}(J) t \leq \ell(\gamma)$.

By i)

$$
\left|\left\{q \in \mathbb{H}: \quad d_{c}(p, \gamma)<t\right\}\right| \leq c_{11} \ell(\gamma) t^{3} .
$$

Replacing (4.7) in (4.6) we get

$$
\int_{\mathbb{H}} \int_{0}^{1} \beta_{\mathbb{H}}^{2}(p, t) d p \frac{d t}{t^{4}} \leq c_{12} l(\gamma) \int_{0}^{1} t^{\alpha-1} d t=c_{13} \ell(\gamma) .
$$

In fact we showed above that $\gamma$ is chord-arc (i.e.) $d_{c}(\gamma(t), \gamma(\theta)) \geq c_{0}$ $t-\theta \mid$, for $t, \theta \in[0, T]$, and that

$$
\int_{\mathbb{H}} \int_{0}^{1} \beta_{\mathbb{H}}^{2}(p, t) d p \frac{d t}{t^{4}} \leq c\left(c_{0}\right) \ell(\gamma) .
$$

In particular we have 
Corollary 4.1. If $\gamma$ is a minimizing geodesic, then

$$
\int_{\mathbb{H}} \int_{0}^{1} \beta_{\mathbb{H}}^{2}(p, t) d p \frac{d t}{t^{4}} \leq C \ell(\gamma),
$$

where $C$ is an absolute constant.

Corollary 4.2. Let $\gamma$ be a finite union of $\mathbf{C}^{1, \alpha}$-regular simple horizontal curves $\gamma_{1}, \ldots, \gamma_{N}$. Then,

$$
\int_{\mathbb{H}} \int_{0}^{1} \beta_{\mathbb{H}}^{2}(p, t) d p \frac{d t}{t^{4}}<+\infty .
$$

Proof. It is enough to notice that

$$
\sup _{p \in \gamma \cap U_{c}\left(p_{0}, t\right)} \frac{d_{c}(p, r)}{t} \leq \sum_{j=1}^{N} \sup _{q \in \gamma_{j} \cap U_{c}\left(p_{0}, t\right)} \frac{d_{c}(p, r)}{t} .
$$

Remark 4.1. It is evident from the proof of previous theorem that the Hölder regularity assumption (2) could be weakened by requiring only a Dini-type condition for $\gamma^{\prime}$ as follows: denote by $\sigma$ the modulus of continuity of $\gamma^{\prime}$ (i.e. let $\sigma$ be such that $\left|\gamma^{\prime}(t)-\gamma^{\prime}(s)\right| \leq \sigma(|t-s|)$ for $\left.t, s \in[0, T]\right)$. Then

$$
\int_{0}^{1} \frac{\sigma(t)}{t} d t<\infty
$$

\section{Appendix: Rectifiable curves in metric spaces}

In this appendix, we collect some results we used in the special setting of the Heisenberg group. Throughout all this section, $(X, d)$ is a metric space.

\subsection{Rectifiable curves in metric spaces}

A curve $\gamma$ is a continuous map $\gamma: I \rightarrow X$ (or is its image) where $I \subset \mathbb{R}$ is an interval. If $\gamma$ is an injective map, we say that $\gamma$ is a Jordan curve. If $\gamma$ is a Lipschitz map, we say that $\gamma$ is a Lipschitz curve. For simplicity, we assume that $I$ is a compact interval, that is of the form $I=[a, b]$, where $a<b$. The length of the curve $\gamma: I \rightarrow X$ is given by

$$
\ell(\gamma)=\sup \sum_{i=1}^{N} d\left(\gamma\left(t_{i}\right), \gamma\left(t_{i-1}\right)\right)
$$

where the supremum is taken over all subdivisions $t_{0}=a<t_{1}<\ldots t_{N-1}<$ $t_{N}=b$ of $[a, b]$. 
A curve $\gamma$ is said to be rectifiable if its length is finite. For instance, a Lipschitz curve is rectifiable and its length is less than $M|a-b|$ (where $M$ is the Lipschitz constant of $\gamma$ ) . For any rectifiable curve, there are its associated length functions $s: I \rightarrow[0, \ell(\gamma)]$ and a unique 1-Lipschitz map $\gamma_{s}:[0, \ell(\gamma)] \rightarrow X$ such that $\gamma=\gamma_{s} \circ s$. The map $\gamma_{s}$ is called the arc-length parametrization of $\gamma$.

We define the metric derivative of $\gamma$ at the point $x \in I$ as the limit (if it exists)

$$
\lim _{t \rightarrow 0} \frac{d(\gamma(x+t), \gamma(x))}{|t|}
$$

We denote by $|\dot{\gamma}|(x)$ this limit (whenever this limit exists). For each Lipschitz curve $\gamma:[a, b] \rightarrow X$, the metric derivative exists almost everywhere. Moreover, $\ell(\gamma)=\int_{a}^{b}|\dot{\gamma}|(t) d t$ (see [2] for more details).

\subsection{Hausdorff measures and Hausdorff distance}

Let $A$ be a subset of $X$. We define its 1 -Hausdorff measure $\mathcal{H}^{1}(A)$ by

$$
\mathcal{H}^{1}(A)=\lim _{\delta \rightarrow 0}\left(\inf \left\{\sum_{i \in I}\left(\operatorname{diam} A_{i}\right) ; \operatorname{diam} A_{i}<\delta, A \subset \cup_{i \in I} A_{i}\right\}\right) .
$$

We now recall basic things about Hausdorff distance (see [10], [2], [8] for more details). Let $A, B$ be two subsets of the metric space $(X, d)$. We define the Hausdorff distance between $A$ and $B$ by

$$
d_{H}(A, B)=\inf \left\{r>0, A \subset U_{r}(B) \text { and } B \subset U_{r}(A)\right\}
$$

where $U_{r}(S)$ is the $r$-neighborhood of $S$, that is $U_{r}(S)=\{x \in X ; d(x, S)<r\}$ (or equivalently $U_{r}(S)=\bigcup_{x \in S} B(x, r)$ ).

Let $\mathcal{M}(X)$ be the set of closed subsets of $X$. Then, if $X$ is compact, $\mathcal{M}(X)$ equiped with the Hausdorff distance is compact.

A sequence of closed sets $\left(K_{n}\right)$ in the metric space $(X, d)$ is said to converge in the Kuratowski sense to a closed set $K$ if the following conditions hold:

(i) If $x=\lim _{k \rightarrow+\infty} x_{n_{k}}$ for some subsequence $\left(x_{n_{k}}\right)$ of a sequence $\left(x_{n}\right)$ such that $x_{n} \in K_{n}$ for any $n$, then $x \in K$;

(ii) If $x \in K$, then there exists a sequence $\left(x_{n}\right)$ such that $x_{n} \in K_{n}$ for any $n$ and $x=\lim _{n \rightarrow+\infty} x_{n}$.

In fact, if the sequence of closed sets $\left(K_{n}\right)$ converges to the closed set $K$ with respect to the Hausdorff distance, then $\left(K_{n}\right)$ converges to $K$ in the sense of Kuratowski. Moreover, if the space $X$ is compact, then the converse is true. 
The classical Golab theorem states that if $\left(K_{n}\right)$ is a sequence of compact connected sets in $X$ which converges to $K$ (in the Hausdorff sense), then $K$ is connected and $\mathcal{H}^{1}(K) \leq \liminf _{n \rightarrow+\infty} \mathcal{H}^{1}\left(K_{n}\right)$. From all these results, we get the following statement.

Theorem 5.1. Let $X$ be a proper metric space and let $E$ be a compact, closed subset of $X$. Let $\left(x_{n}^{j}\right)_{j \in \mathbb{Z}, n=1, \ldots, N_{j}}$ be a net associated with $E$ and let $\left(\Gamma_{j}\right)$ be a sequence of continua such that diam $\Gamma_{j}$ are uniformly bounded, and, for any $j \in \mathbb{Z}$, any $n=1, \ldots, N_{j}, x_{n}^{j} \in \Gamma_{j}$. Then there exists a Hausdorff-convergent subsequence of $\left(\Gamma_{j}\right)$. Moreover, if we denote by $\Gamma$ the limit of this subsequence $\left(\Gamma_{n_{k}}\right), \Gamma$ is a continuum which contains $E$ and

$$
\mathcal{H}^{1}(\Gamma) \leq \liminf _{k \rightarrow+\infty} \mathcal{H}^{1}\left(\Gamma_{n_{k}}\right)
$$

Recall that a continuum $K$ in $X$ is a compact, connected subset of $X$ which contains more than one point and that a metric space $(X, d)$ is proper if closed balls are compact in $X$. The fact that $\left(x_{n}^{j}\right)_{j \in \mathbb{Z}, n=1, \ldots, N_{j}}$ is a net of $E$ means that, for any $j \in \mathbb{Z}$,

(i) $d\left(x_{n}^{j}, x_{m}^{j}\right)>2^{-j}$ if $n \neq m$.

ii) For any $x \in E$, there exists $n \in\left\{1, \ldots, N_{j}\right\}$ such that $d\left(x, x_{n}^{j}\right) \leq 2^{-j}$.

\subsection{Geodesic spaces}

We say that $\gamma:[a, b] \rightarrow X$ is a shortest path (connecting $x$ and $y$ ) if $\gamma(a)=x$, $\gamma(b)=y$ and $l(\gamma)=d(x, y)$. A curve $I \rightarrow X$ is geodesic if for every $t \in I$, there exists a sub-interval $J$ of $I$ containing a neighborhood of $t$ in $I$ such that the restriction of $\gamma$ to $J$ is a shortest path. In other words, a geodesic is a curve which is locally a shortest path. A curve $\gamma: I \rightarrow X$ is a minimal geodesic if its restriction to any interval $J \subset I$ is a shortest path. We say that $X$ is a geodesic space if any pair of points $x, y$ in $X$ can be connected by a shortest path.

The next result says that a continuum with finite 1-Hausdorff measure is almost a rectifiable curve.

Proposition 5.2. Let $X$ be a doubling geodesic space and let $E$ be a continuum in $X$ with $\mathcal{H}^{1}(E)<+\infty$. Then, there exists a Lipschitz curve $\Gamma=\gamma([0, L])$ in $X$ such that

(i) $E \subset \Gamma$;

(ii) $\mathcal{H}^{1}(E) \leq L \leq C \mathcal{H}^{1}(E)$ where $C>0$ depends only the doubling constant $C_{d}$ of $X$

(iii) $|\dot{\gamma}|(t)=1$ for almost every $t \in[0, L]$. 
Note that $L$ is comparable to $\ell(\Gamma)$. Hence, $C^{-1} \mathcal{H}^{1}(E) \leq \ell(\Gamma) \leq C \mathcal{H}^{1}(E)$. Recall that $X$ is a doubling metric space if there exists $C_{d} \in \mathbb{N} \backslash\{0\}$ such that for any $x \in X$, any $R>0$, the open ball $B(x, R)$ can be covered by at most $C_{d}$ open balls of radius $R / 2$. A typical example of doubling geodesic space is the Heisenberg group $\mathbb{H}$ equipped with its Carnot-Carathéodory metric $d_{c}$.

Proof. We adapt the proof of Theorem 1.8 of [9]. This argument is based on the following classical result of graph theory, which can be proved by induction on the number of edges.

Lemma 5.3. Let $G$ be a connected graph with only finitely many edges. Then there is a path that traverses each edge exactly twice (once in each direction).

Without loss of generality, we assume that $\operatorname{diam} E=1$. For any $\delta \in(0,1)$, consider a set $A_{\delta}$ of points of $E$ such that

(P1) For any $x, y \in A_{\delta}, x \neq y, d(x, y) \geq 2 \delta$.

(P2) For any $x \in E$, there exists $y \in A_{\delta}$ such that $d(x, y)<2 \delta$.

Note that, since the metric space $(X, d)$ is doubling, $A_{\delta}$ is finite for any $\delta \in(0,1)$. Now, we construct a graph $E_{\delta}$ associated with $A_{\delta}$ in the following way. The vertices of $E_{\delta}$ are points of $A_{\delta}$ and two vertices $x$ and $y$ are joined by an edge if $d(x, y) \leq 4 \delta$. The graph $E_{\delta}$ could be also seen as a subset $\Delta_{\delta}$ of $X$. Indeed, the set $\Delta_{\delta}$ is obtained by joining, for any edge $(x, y)$ of $E_{\delta}$, the corresponding points of $A_{\delta}$ by a geodesic segment, denoted by $[x, y]$. The graph $E_{\delta}$ has finitely many edges. In order to apply Lemma 5.3, we should prove that $E_{\delta}$ is also connected. Assume that this is not the case, that is we can write $E_{\delta}=Y \cup Z$ where $Y$ and $Z$ are disjoint, nonempty, and satisfy $d(y, z)>4 \delta$ for any $y \in Y$ and any $z \in Z$. Consider now

$$
A_{1}=E \cap\left(\cup_{y \in Y} \overline{B(x, 2 \delta)}\right) \quad \text { and } \quad A_{2}=E \cap\left(\cup_{y \in Z} \overline{B(x, 2 \delta)}\right) .
$$

Then, $A_{1}$ and $A_{2}$ are nonempty, closed and disjoint. Moreover, $A=A_{1} \cup A_{2}$ by property $(\mathrm{P} 2)$ above. This contradicts the fact that $E$ is connected. Hence, for any $\delta \in(0,1), E_{\delta}$ is connected. Moreover, we claim that $\mathcal{H}^{1}\left(\Delta_{\delta}\right) \leq$ $C \mathcal{H}^{1}(E)$. This follows from these two observations. Let $N_{\delta}$ the number of points of $A_{\delta}$. Then, we have

1) Since $X$ is doubling, the number of neighbours in $E_{\delta}$ of a point of $A_{\delta}$ is bounded uniformly in $\delta$. Thus, $\mathcal{H}^{1}\left(\Delta_{\delta}\right) \leq C \delta N_{\delta}$ (where $C>0$ does not depend on $\delta$ ).

2) Since $E$ is connected, $\mathcal{H}^{1}(E \cap B(x, \delta)) \geq \delta$. To see this, fix $x \in E$ and $r<(\operatorname{diam} E) / 2$. Set $\phi(y)=d(x, y)$ for any $y \in E$. It is clear that $\phi$ is 1-Lipschitz. 
Thus, we get

$$
\mathcal{H}^{1}(\phi(E \cap B(x, r))) \leq \mathcal{H}^{1}(E \cap B(x, r)) .
$$

But, $H^{1}$ on $\mathbb{R}$ is an outer Lebesgue measure. Hence, since $\phi(E \cap B(x, r))$ is an interval of the form $[0, a]$, we also have

$$
\mathcal{H}^{1}(\phi(E \cap B(x, r)))=\sup _{y, z \in B(x, r)}|d(x, y)-d(y, z)|=\sup _{y \in B(x, r)} d(x, y) .
$$

Since $E$ is connected, by continutiy of the metric $d, \sup _{y \in B(x, r)} d(x, y) \geq r$ and then

$$
\mathcal{H}^{1}(E \cap B(x, r)) \geq \mathcal{H}^{1}(\phi(E \cap B(x, r))) \geq r .
$$

Moreover, the $E \cap B(x, \delta), x \in A_{\delta}$, are disjoint. Thus, $\mathcal{H}^{1}(E) \geq N_{\delta} \delta$ and then $\mathcal{H}^{1}\left(\Delta_{\delta}\right) \leq C \mathcal{H}^{1}(E)$.

We can now apply Lemma 5.3 to $\Delta_{\delta}$. Using the path given by Lemma 5.3 , we can easily construct a Lipschitz mapping (and hence a Lipschitz curve) $\gamma_{\delta}:[0,1] \rightarrow \Delta_{\delta}$ such that $\gamma_{\delta}([0,1])=\Delta_{\delta}$ and $\left\|\left|\dot{\gamma}_{\delta}\right|\right\|_{\infty} \leq C \mathcal{H}^{1}(E)$. Recall that $\left|\dot{\gamma}_{\delta}\right|$ is the metric derivative of $\gamma_{\delta}$. Now, by the Arzelà-Ascoli theorem, we find a sequence $\delta_{j}$ which converges to 0 and such that the $\gamma_{\delta_{j}}$ converge uniformly on $[0,1]$. The limit function $\tilde{\gamma}:[0,1] \rightarrow X$ is Lipschitz continuous, with Lipschitz norm $\leq C \mathcal{H}^{1}(E)$ and such that $\tilde{\gamma}([0,1])=E$. We get the desired Lipschitz function $\gamma$ by applying the following reparametriation result (see [2] for a proof).

Theorem 5.4. Let $\phi:[a, b] \rightarrow X$ be a Lipschitz mapping and let $L$ be its total variation (or equivalently the length of its range). Then there exists a Lipschitz curve $\tilde{\phi}:[0, L] \rightarrow X$ such that $|\dot{\tilde{\phi}}|=1$ almost everywhere on $[0, L]$ and $\tilde{\phi}(0, L)=\phi([a, b])$.

\subsection{Menger curvature and rectifiable curves}

If $x, y$ and $z$ are three pairwise distinct points in the metric space $(X, d)$, a comparison triangle of the triple $(x, y, z)$ is a triangle with vertices $\bar{x}, \bar{y}, \bar{z}$ in the Euclidean plane $\mathbb{R}^{2}$ such that

$$
d(x, y)=|\bar{x}-\bar{y}|, d(y, z)=|\bar{y}-\bar{z}|, d(z, x)=|\bar{z}-\bar{x}|
$$

where $|.-$.$| denotes the Euclidean metric in \mathbb{R}^{2}$.

Let $x, y$ and $z$ be three pairwise disjoint points of the metric space $(X, d)$. The Menger curvature of the triple $(x, y, z)$ is defined by

$$
c(x, y, z)=2 \frac{\sin \theta(\bar{x} \bar{z} \bar{y})}{|\bar{x}-\bar{y}|}
$$


where $\bar{x} \bar{y} \bar{z}$ is a comparison triangle of $(x, y, z)$ and where $\theta(\bar{x} \bar{z} \bar{y})$ denotes the angle at $\bar{z}$ of the triangle with vertices $\bar{x}, \bar{y}, \bar{z}$. By elementary geometry, we get

$$
c(x, y, z)=\frac{4 S(\bar{x}, \bar{y}, \bar{z})}{|\bar{x}-\bar{y}||\bar{y}-\bar{z}||\bar{z}-\bar{x}|}
$$

where $S(\bar{x}, \bar{y}, \bar{z})$ denotes the area of the Euclidean triangle with vertices $\bar{x}, \bar{y}$ and $\bar{z}$. Therefore,

$$
c(x, y, z)=\frac{2 \sin \theta(\bar{x} \bar{y} \bar{z})}{|\bar{x}-\bar{z}|}=\frac{2 \sin \theta(\bar{y} \bar{x} \bar{z})}{|\bar{y}-\bar{z}|} .
$$

Moreover,

$$
c(x, y, z)=\frac{2 d\left(\bar{x}, L_{\bar{y}, \bar{z}}\right)}{|\bar{x}-\bar{y}||\bar{x}-\bar{z}|}
$$

where $L_{\bar{y}, \bar{z}}$ is the line in $\mathbb{R}^{2}$ passing through $\bar{y}$ and $\bar{z}$.

Let $E \subset X$. If $p \in E$, we define the Menger curvature of $E$ at $p$ (denoted by $\left.c_{E}(p)\right)$ by (if the limit exists)

$$
c_{E}(p)=\lim _{q, r, s \in E \rightarrow p} c(q, r, s) .
$$

In the book of Blumenthal and Menger [7] is proved the following result.

Theorem 5.5. Let $E$ be a continuum in the metric space $(X, d)$.

(i) If $c_{E}(p)<+\infty$ for $p \in E$, then in a neighborhood of $p, E$ is a rectifiable Jordan curve.

(ii) If $c_{E}(p)<+\infty$ for all $p \in E$, then $E$ is a rectifiable Jordan curve.

From Theorem 2, we easily get the followings results.

Proposition 5.6. Let $X$ be a geodesic metric space and let $E$ be a continuum in $X$. Then,

(i) $E$ is a geodesic curve if and only if $c_{E}(x)=0$ for every $x \in E$.

(ii) $E$ is a minimal geodesic curve if and only if $c(x, y, z)=0$ for all $x, y$ and $z$ in $E$.

Before giving Hahlomaa's version of the geometric traveling theorem in metric spaces, we need to define $\beta$ numbers in this general setting. Let $E \subset X$ be a compact set and let $C_{1} \geq C_{2}$ large enough. For any $x \in X$ and any $t>0$, set

$\beta_{X}(x, t)=\sup \left\{c\left(z_{1}, z_{2}, z_{3}\right): z_{1}, z_{2}, z_{3} \in E \cap B(x, t), d\left(z_{i}, z_{j}\right) \geq C_{1}^{-1} t, \forall i \neq j\right\}$. 
Recall that $\Delta=\left(\Delta_{k}\right)_{k \in \mathbb{Z}}$ is a net of $E$ if $\Delta_{k}, k \in \mathbb{Z}$, are subsets of $E$ such that

(i) For any $x, y \in \Delta_{k}$ with $x \neq y, d(x, y)>2^{-k}$.

(ii) For any $x \in E$, there exists $y \in \Delta_{k}$ such that $d(x, y) \leq 2^{-k}$.

Set

$$
\beta_{X}(E)=\inf \left\{\sum_{k \in \mathbb{Z}} \sum_{x \in \Delta_{k}} \beta_{X}^{2}\left(x, C_{2} 2^{-k}\right)\left(2^{-k}\right)^{3} ;\left(\Delta_{k}\right) \text { is a net of } E\right\} .
$$

Theorem 5.7. ([16]) Let $E$ be a compact subset of the metric space $(X, d)$. If $\beta_{X}(E)<+\infty$, then $E$ is contained in a rectifiable curve $\Gamma$. Moreover, there exists an absolute constant $C>0$ (which does not depend on $E$ ) such that $\inf _{\Gamma \supset E} l(\Gamma) \leq C\left(\beta_{X}(E)+\operatorname{diam} E\right)$.

It should be noted that in a previous version of this paper, we proved Theorem 5.7 under the extra assumptions that the metric space $X$ is doubling and geodesic. Our construction was similar to the one given in Section 3. In [23], comparison results between Euclidean $\beta$ numbers and Menger curvature are given. In fact, in the Euclidean setting, $\beta$ numbers are easier to work with than the Menger curvature. In the special case $(X, d)=\left(\mathbb{R}^{n}, d_{\text {Euc }}\right)$, Hahlomaa's condition on the $\beta$ 's is equivalent to Jones'one.

\section{References}

[1] Ambrosio, L., Rigot, S.: Optimal mass transportation in the Heisenberg group. J. Funct. Anal. 208 (2004), no. 2, 261-301.

[2] Ambrosio, L., Tilli, P.: Topics on analysis in metric spaces. Cambridge University Press, 2004.

[3] Bellaïche, A.: The tangent space in subriemannian geometry. In Subriemannian Geometry, 1-78. Progr. Math. 144. Birkhäuser, Basel, 1996.

[4] Bishop, C., Jones, P.: Harmonic measure, $L^{2}$ estimates and the Schwarzian derivative. J. Anal. Math. 62 (1994), 77-113.

[5] Bishop, C., Jones, P.: Hausdorff dimension and Kleinian groups. Acta Math. 179 (1997), no. 1, 1-39.

[6] Bishop, C., Jones, P., Pemantle, R., Peres, Y.: The dimension of the Brownian frontier is greater than 1. J. Funct. Anal. 43 (1997), no. 2, 309-336.

[7] Blumenthal, L. M., Menger, K.: Studies in geometry. W. H. Freeman and Company, San Francisco, 1970. 
[8] Burago, D., Burago, Y., Ivanov, S.: A course in metric geometry. Graduate Studies in Mathematics 33. American Mathematical Society, Providence, 2001.

[9] David, G., Semmes, S.: Analysis of and on uniformly rectifiable sets. Mathematical Surveys and Monographs 38. American Mathematical Society, Providence, 1993.

[10] Federer, H.: Geometric measure theory. Die Grundlehren der mathematischen Wissenschaften 153. Springer-Verlag, New York, 1969.

[11] Franchi, B., Serapioni, R., Serra Cassano, F.: Rectifiability and perimeter in the Heisenberg group. Math. Ann. 321 (2001), no. 3, 479-531.

[12] Franchi, B., Serapioni, R., Serra Cassano, F.: Regular submanifolds, graphs and area formula in Heisenberg groups. Adv. Math. 211 (2007), 152-203.

[13] Gaveau, B.: Principe de moindre action, propagation de la chaleur et estimées sous elliptiques sur certains groupes nilpotents. Acta Math. 139 (1977), no. 1-2, 95-153.

[14] Graczyk, J., Jones, P.: Dimension of the boundary of quasi-conformal Siegel disks. Invent. Math. 148 (2002), no. 3, 465-493.

[15] Gromov, M.: Metric structures for Riemannian and non-Riemannian spaces. Progress in Mathematics 152. Birkhäuser, Boston, MA, 1999.

[16] HahlomaA, I.: Menger curvature and Lipschitz parametrizations in metric spaces. Fund. Math. 185 (2005), no. 2, 143-169.

[17] Jones, P.: Square functions, Cauchy integrals, analytic capacity, and harmonic measure. In Harmonic analysis and partial differential equations, 24-68. Lecture Notes in Mathematics 1384. Springer-Verlag, 1989.

[18] Jones, P.: Rectifiable sets and the traveling salesman problem. Invent. Math. 102 (1990), no. 1, 1-15.

[19] Mattila, P.: Geometry of sets and measures in Euclidean spaces. Cambridge Studies in Advanced Mathematics 44. Cambridge University Press, Cambridge, 1995.

[20] Mitchell, J.: On Carnot-Carathéodory metrics. J. Differential Geom. 21 (1985), no. 1, 35-45.

[21] Okikiolu, K.: Characterizations of subsets of rectifiable curves in $\mathbb{R}^{n}$. J. London Math. Soc. (2) 46 (1992), no. 2, 336-348.

[22] Pajot, H.: Sous-ensembles de courbes Ahlfors-régulières et nombre de Jones. Publ. Mat. 40 (1996), no. 2, 497-526.

[23] Pajot, H.: Analytic capacity, rectifiability, Menger curvature and the Cauchy integral. Lecture Notes in Mathematics 1799. Springer Verlag, Berlin, 2002.

[24] Pansu, P.: Géométrie du Groupe d'Heisenberg. Thèse pour le titre de Docteur de 3ème cycle, Université Paris VII, 1982. 
[25] Pansu, P.: Une inégalité isopérimétrique sur le groupe de Heisenberg. C. R. Acad. Sci. Paris Sér. I Math. 295 (1982), no. 2, 127-130.

[26] Schul, R.: Subset of rectifiable curves in Hilbert space and the analyst's TSP. PhD dissertation, Yale University, 2005.

[27] Stein, E. M.: Harmonic Analysis: real-variable methods, orthogonality, and oscillatory integrals. Princeton Mathematical Series 43. Princeton University Press, Princeton, 1993.

[28] Varopoulos, N. Th., Saloff-Coste, L., Coulhon, T.: Analysis and Geometry on Groups. Cambridge Tracts in Mathematics 100. Cambridge University Press, Cambridge, 1992.

Recibido: 30 de marzo de 2005

Fausto Ferrari

Dipartimento di Matematica dell'Università

Piazza di Porta S. Donato, 5

40126 Bologna, Italy

and

C.I.R.A.M.

Via Saragozza 8

40133 Bologna, Italy

ferrari@dm.unibo.it

Bruno Franchi

Dipartimento di Matematica dell'Università

Piazza di Porta S. Donato, 5

40126 Bologna, Italy

franchib@dm. unibo.it

Hervé Pajot

Université de Grenoble I

Institut Fourier, Laboratoire de Mathématiques

UMR 5582, BP 74

38402 Saint Martin d'Hères Cédex, France

herve.pajot@ujf-grenoble.fr

F.F. and B.F. were supported by University of Bologna, Italy, funds for selected research topics and by GNAMPA of INdAM, Italy, under project "Analysis in metric spaces and subelliptic equations". H.P. is partially supported by the European commission (European TMR Network "Harmonic Analysis and Related Problems"). Part of this work has been done during a stay of the third author at the University of Bologna. He would like to thank this institution for its warm hospitality. 